\title{
Structures of the human Mediator and Mediator-bound preinitiation complex
}

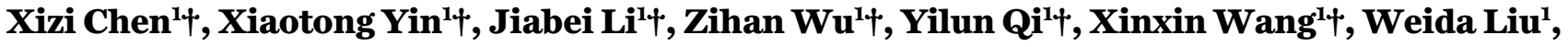 \\ Yanhui $\mathrm{Xu}^{1,2,3 *}$
}

${ }^{1}$ Fudan University Shanghai Cancer Center, Institutes of Biomedical Sciences, State Key Laboratory of Genetic Engineering, Shanghai Key Laboratory of Radiation Oncology, and Shanghai Key Laboratory of Medical Epigenetics, Shanghai Medical College of Fudan University, Shanghai 200032, China. ${ }^{2}$ International Colaboratory of Medical Epigenetics and Metabolism, Ministry of Science and Technology, China, Department of Systems Biology for Medicine, School of Basic Medical Sciences, Shanghai Medical College of Fudan University, Shanghai 200032, China. ${ }^{3}$ Human Phenome Institute, Collaborative Innovation Center of Genetics and Development, School of Life Sciences, Fudan University, Shanghai 200433, China.

†These authors contributed equally to this work.

${ }^{*}$ Corresponding author. Email: xuyh@fudan.edu.cn

The 1.3-MDa transcription factor IID (TFIID) is required for preinitiation complex (PIC) assembly and RNA polymerase II (Pol II)-mediated transcription initiation on almost all genes. The 26-subunit Mediator stimulates transcription and cyclin-dependent kinase 7 (CDK7)-mediated phosphorylation of Pol II Cterminal domain (CTD). We determined the structures of human Mediator in the Tail module-extended (at near-atomic resolution) and Tail-bent conformations and structures of TFIID-based PIC-Mediator (76 polypeptides, $\sim 4.1 \mathrm{MDa}$ ) in four distinct conformations. PIC-Mediator assembly induces concerted reorganization (Head-tilting and Middle-down) of Mediator and creates a Head-Middle sandwich, which stabilizes two CTD segments and brings CTD to CDK7 for phosphorylation, suggesting a CTD-gating mechanism favorable for phosphorylation. The TFIID-based PIC architecture modulates Mediator organization and TFIIH stabilization, underscoring the significance of TFIID in orchestrating PIC-Mediator assembly.

The RNA polymerase II (Pol II)-mediated eukaryotic transcription initiation starts with the assembly of a preinitiation complex (PIC) (1-3). Stepwise PIC assembly involves core promoter recognition by the transcription factor IID (TFIID), which is formed by TATA box-binding protein (TBP) and 13 TBP-associated factors (TAF1-TAF13) (4-6). TFIID recruits Pol II and other general transcription factors (GTFs) TFIIA, TFIIB, TFIIF, TFIIE, and TFIIH to assemble a holo-PIC, which we termed hPIC or TFIID-based PIC, in contrast to the extensively studied TBP-based PIC (PIC ${ }^{\mathrm{TBP}}$ ). TFIID is globally required for almost all Pol II-mediated transcription initiation and could not be replaced by TBP (6-9).

TFIIH consists of a seven-subunit core module and a cyclin-dependent kinase (CDK)-activating kinase module (CAK) formed by CDK7, cyclin $\mathrm{H}(\mathrm{CycH})$, and MAT1 (10-13). CDK7 phosphorylates $S^{5}$ residues of the heptapeptide repeats $\left(\mathrm{Y}^{1} \mathrm{~S}^{2} \mathrm{P}^{3} \mathrm{~T}^{4} \mathrm{~S}^{5} \mathrm{P}^{6} \mathrm{~S}^{7}\right)$ of the C-terminal domain (CTD) of RPB1, the largest subunit of Pol II (10-12). The multisubunit Mediator complex is required for Pol II-mediated eukaryotic transcription (14-16). As a critical transcription coactivator, Mediator binds Pol II, transduces regulatory signals from enhancer- or promoter-bound transcription factors (TFs) to Pol II, facilitates PIC assembly, stimulates CDK7-mediated CTD phosphorylation, and is involved in higher order chromatin organization (17-23). The critical role of Mediator in transcription is further underscored by the dysregulation of various Mediator subunits in human diseases $(24,25)$.

The Mediator (MED) complexes in yeast (up to 25 subunits) and humans (30 subunits) share a generally similar modular composition and are organized by a dissociable 4subunit kinase module and a core complex formed by Head, Middle, and Tail modules (26). Structures of Mediator have been extensively studied (26-36) and mouse Mediator structures were recently reported $(37,38)$. Previous studies also reported structures of $\mathrm{PIC}^{\mathrm{TBP}}(39,40)$, $\mathrm{PIC}^{\mathrm{TBP}}$ bound to core Mediator (PIC ${ }^{\mathrm{TBP}}$-cMED) (40), PIC ${ }^{\mathrm{TBP}}-\mathrm{MED}(41,42)$, and Pol II-MED from yeast $(27,28,43)$ and thermophilic fungus (44). We have recently reported cryo-electron microscopy (EM) structures of TFIID-based PIC in distinct assembly states (45). Despite these studies, it remains elusive how Mediator is assembled into TFIID-based PIC and regulates Pol II CTD phosphorylation. 


\section{Structure determination of human Mediator}

We purified the $\sim 1.5$-MDa human Mediator to homogeneity through co-transfection of 26 Mediator subunits into human embryonic kidney Expi293F cells, followed by immunoprecipitation and ion-exchange (fig. S1). The Mediator kinase module inhibits the assembly of Mediator into PIC and was not included (46). The presence of 26 subunits in the purified complex was confirmed by mass spectrometry and structural determination. The purified recombinant Mediator stimulated transcription initiation in an in vitro transcription assay, indicative of a functional complex (fig. S1E).

The structure of Mediator was determined to a nominal resolution of $4.0 \AA \AA$ using cryo-EM single particle reconstruction (figs. S2, A to F, and S3F). The deletion of MED1 intrinsically disordered regions (IDR) improved the complex homogeneity (fig. S1C). The cryo-EM map of Mediator ${ }^{\triangle \mathrm{MEDI}-\mathrm{IDR}}$ (termed Mediator for simplicity) was increased to $3.5 \AA$ resolution and the focused refinement largely improved the quality of cryo-EM maps of the Tail (3.2 $\AA$ ), the Head (2.8 $\AA$ ), and the Middle (3.7 ̊̊) modules (Fig. 1, B to E, fig. S2, G to $\mathrm{O}$, and movies S1 to S8). The wild-type Mediator (Mediator $^{\mathrm{WT}}$ ) and Mediator ${ }^{\mathrm{MEDD}-\mathrm{IDR}}$ showed almost identical fold (fig. S3, A and F) and comparable stimulation in basal transcription activity (fig. S1E).

Structural model was built into the cryo-EM maps with structures of yeast Mediator (yMed) (29) and human MED23 (47) as templates (Fig. 1F, fig. S3, table S1, and data S1). All of the 26 subunits were traced with the majority of residues being unambiguously modeled and a few segments being built with poly-alanine chains. Model building was also aided by secondary structure prediction (data S2) and crosslinking mass spectrometry (XL-MS) analyses (fig. S4).

\section{Overall structure of Mediator}

The structure reveals an elongated fold with approximate dimensions of $\sim 350 \times 200 \times 150 \AA^{3}$ (Fig. 1, B and F, fig. S5, and movie S1). The previously defined (37) Head, Middle, and Tail modules were clearly visualized. The Head and Middle are separated at the top and merge with the Tail at the bottom. Two scaffold subunits, MED14 and MED17, involve the formation of the Middle, Head, and upper part of the Tail (termed Shoulder) (Fig. 1, A to D, fig. S6, and movies S2 and S5).

The Head and Shoulder (Head-Shoulder) is organized into a three-stranded super-scaffold, in which MED17 (central strand) is sandwiched by MED14 (inner strand) and a 9subunit THB (tandem $\alpha$-helix bundles) submodule (outer strand) (Fig. 1, A to C, fig. S7, and movie S2). Three sequentially associated subunits (MED18, MED20, and MED27) bind and stabilize the super-scaffold (Fig. 1F and fig. S7). The Tail adopts an extended conformation and could be divided into the Shoulder and a two-branched Saddle (Fig. 1,
B and E, fig. S8, and movie S3). The two branches are located below the Head-Shoulder and Middle, and are termed Hbranch and M-branch, respectively. The Middle adopts an arch-shaped conformation and is divided into the Hook, Knob, Rod, and Elbow (Fig. 1D, fig. S9, and movie S4). The Hook and Rod together form a kinked long helix bundle and the Elbow at the bottom bridges the Middle and Tail. The Knob and the associated metazoan-specific subunit MED26 are positioned at the turning point of the Middle (supplementary text).

\section{MED14 and MED17 are scaffold subunits in nucleating Mediator}

As the central scaffold subunit of Mediator, MED14 spans over $\sim 270 \AA$ and makes extensive intermolecular contacts with 14 Mediator subunits throughout the Middle, Head, and Shoulder modules (Fig. 2, A to D, fig. S6, movies S2, S4, S5, and S6, and supplementary text). The major portion of MED14 exists in the Head-Shoulder and consists of nine tandemly arrayed repetitive $\alpha \beta$ motifs $\left(\mathrm{RM}^{\mathrm{MED1}}{ }^{\mathrm{M}}\right.$ to RM9 ${ }^{\mathrm{MED14}}$ ), which are arranged in an atypical right-handed helix with $\sim 4$ RMs per turn. MED17 serves as another scaffold and binds 15 Mediator subunits around the HeadMiddle interface and in the Head-Shoulder. The major portion of MED17 consists of four tandemly arrayed RMs (RM1 to RM4). The scaffolding roles of MED14 and MED17 agree with their critical roles in Mediator assembly and function $(27,35,48-51)$.

\section{The THB nucleates the Head-Shoulder}

The THB is formed by five $\alpha$-helix bundles (HB1 to HB5), which are tandemly arrayed into an extended zigzag scaffold (Fig. 2, C to E, fig. S7, movies S2 and S6, and supplementary text). Within the THB, two successive $\alpha$-helix bundles are connected by at least two subunits: HB1-HB2 by MED8 and MED17; HB2-HB3 by MED11 and MED22; HB3-HB4 by MED28 and MED30; and HB4-HB5 by MED27 and MED29. Each of these connecting subunits possesses two $\alpha$-helices that are incorporated into the two connecting $\alpha$-helix bundles.

The HB1 and HB2 are in parallel and form a rigid HB1HB2 core (previously termed Neck domain) $(29,52)$, which is movable relative to the rest of the Head-Shoulder (Fig. 2, $\mathrm{C}$ to E, and fig. S7). The HB1 is capped by the N-terminal $\alpha \beta$ domain of MED6 ( $\alpha \beta^{\mathrm{MED} 6}$ ). The four-helix bundle HB3 nucleates the RM2 and RM3 of MED17, the mixed $\alpha \beta$ domain of MED27 ( $\alpha \beta^{\text {MED27 }}$ ), and MED18-MED20 heterodimer (MED18/20). The HB4 and HB5 in the Shoulder together adopt a V-shaped fold and make extensive contacts with MED17 and MED14. Such modular organization offers the THB both connectivity and flexibility, consistent with its 
essential role in Mediator assembly and binding of PIC (see below).

\section{Organization of the metazoan-specific Saddle}

The Saddle is formed by MED16, MED24, and two metazoan-specific subunits, MED23 and MED25, generating considerable increase in the size and complexity of the Tail (Fig. 3A, fig. S8, and movie S3). MED23 consists of five HEAT repeat regions $\left(\mathrm{HR}^{\mathrm{MED} 23}\right.$ to $\mathrm{HR} 5^{\mathrm{MED} 23}$ ), in which $\mathrm{HR} 2^{\mathrm{MED} 23}$ to $\mathrm{HR} 5^{\mathrm{MED} 23}$ are tandemly arrayed into a triangular core (MED23 core) and $\mathrm{HR1}^{\mathrm{MED} 23}$ protrudes out to bind the $\mathrm{C}$ terminal $\alpha \beta$ domain of the modeled MED15 (MED15 ${ }^{\mathrm{C}}$ ). MED24 also consists of five HEAT repeat regions, in which $\mathrm{HR} 2^{\mathrm{MED} 24}$ to HR5 ${ }^{\mathrm{MED} 24}$ adopt a C-shaped conformation and $\mathrm{HR1}^{\mathrm{MED} 24}$ protrudes out to bind MED15 ${ }^{\mathrm{C}}$ and MED1 (Fig. 3, A to $\mathrm{C}$, and fig. S8, B and E). The $\mathrm{HR}^{\mathrm{MED} 23}$ and $\mathrm{HR} 5^{\mathrm{MED} 23}$ make ridge-to-ridge contacts with the $\mathrm{HR}^{\mathrm{MED} 24}$ and $\mathrm{HR}^{\mathrm{MED} 24}$, generating the M-branch (Fig. 3C). The rest of the two subunits are separated into a Y-shaped base at the bottom of the Saddle. Thus, MED23 and MED24 form a rigid framework of the Tail, in line with their critical roles in Mediator complex assembly $(53,54)$.

MED16 and MED25 together form the H-branch (Fig. 3, $\mathrm{B}$ and D, and fig. S8C). The von Willebrand domain (vWD) of MED25 is partially grasped by MED16 and is in close proximity to $\mathrm{HR}^{\mathrm{MED} 23}$. MED16 has an N-terminal sevenbladed $\beta$-propeller, a central helical bridge, followed by a small $\alpha \beta$ domain $\left(\alpha \beta^{\mathrm{MED} 16}\right)$. The $\beta$-propeller domain fills in the gap of the $\mathrm{C}$-shaped MED24 HEAT repeats, generating a rigid MED16/24 core. The top surface of the $\beta$-propeller is covered by HR $5^{\mathrm{MED} 24}$ and the bottom surface is covered by MED25.

\section{MED1 forms the Elbow and mediates Middle-Saddle contact}

The N-terminal domain of MED1 forms the Elbow of the Middle and binds $\mathrm{HR}^{\mathrm{MED} 24}$ at the M-branch of the Saddle (Fig. 3A and fig. S8E). Three mixed $\alpha \beta$ domains ( $\alpha \beta 1$ to $\alpha \beta 3$ ) are arrayed into a rigid fold and the $\alpha \beta 3$ binds HR1 ${ }^{\text {MED24, }}$ generating the Middle-Saddle contact. The four-helix bundle of the Rod (MED4 and MED9, termed MED4/9) is capped by three short $\alpha$ helices, likely derived from MED1 (fig. S9A). This Rod-Elbow tether serves as a flexible hinge, which coordinates the concerted movements of Mediator upon binding to PIC (see below).

\section{MED15 mediates Shoulder-Saddle contacts}

The Shoulder sits on top of the Saddle and MED15 mediates the Shoulder-Saddle contacts (Fig. 3 and fig. S8D). A short helix hairpin of MED15 (residues 615-655, MED15 ${ }^{\mathrm{N}}$ ) above the $\mathrm{H}$-branch runs into the parallel helices of the exposed HB5 and contacts the $\alpha \beta^{\mathrm{MED} 16}$ (Fig. 3E). The MED15 ${ }^{\mathrm{C}}$ (residues 673 787) above the M-branch is sandwiched by $\mathrm{HRI}^{\mathrm{MED} 23}$ and $\mathrm{HR1}^{\mathrm{MED} 24}$ and binds RM5 ${ }^{\mathrm{MED14}}$ and RM4 $4^{\mathrm{MED17}}$ at the other side (Fig. 3, C and F). An N-terminal loop (residues 14-32) of $\mathrm{HR}^{\mathrm{MED} 23}$ inserts into the gap between MED15 ${ }^{\mathrm{C}}$ and RM4 $4^{\mathrm{MED} 14}$ (fig. S8F). Thus, MED15 serves as a central hub in bridging the Shoulder and Saddle, consistent with the loss of the Tail upon the deletion of MED15 in mouse Mediator (37).

\section{Structure of Mediator in a Tail-bent $\left(\right.$ MED $\left.^{\mathrm{B}}\right)$ conformation}

Cryo-EM 3D classification showed an additional reconstruction of Mediator, in which the Head-Shoulder and Middle remain unchanged whereas the Saddle is flexible and notably bent (Fig. 4, figs. S2 and S10, data S1, and movie S9). The Mediator in the Tail-bent conformation is termed $\mathrm{MED}^{\mathrm{B}}$, in contrast to the abovementioned Mediator structure in a Tail-extended conformation $\left(\mathrm{MED}^{\mathrm{E}}\right)$. The structural model was built by docking $\mathrm{MED}^{\mathrm{E}}$ structural template into the overall map at 4.4 $\AA$ resolution and locally refined cryo-EM map of the Tail at $4.5 \AA$ resolution. No convincing cryo-EM map was observed around MED25 and the helical bridge and $\alpha \beta$ domain of MED16, which were not built in the structural model.

Structural comparison of $\mathrm{MED}^{\mathrm{E}}$ and $\mathrm{MED}^{\mathrm{B}}$ shows considerable conformational differences (from $\mathrm{MED}^{\mathrm{E}}$ to $\mathrm{MED}^{\mathrm{B}}$ ) including a downward rotation of the Saddle, the separation of MED23 core and MED16/24 core at the M-branch, and the dissociation of MED16/24 core from the Head-Shoulder at the H-branch (Fig. 4, fig. S10, and movie S9). Compared to that in $\mathrm{MED}^{\mathrm{E}}, \mathrm{HR1}^{\mathrm{MED} 23}$ of $\mathrm{MED}^{\mathrm{B}}$ moves toward the HeadShoulder by $\sim 25 \AA$ and binds MED15 ${ }^{\mathrm{C}}$ and RM4 $4^{\mathrm{MED14}}$ (Fig. $4 \mathrm{~A})$. The MED23 core rotates downward by $\sim 35^{\circ}$ and moves by as far as $85 \AA$. The $\mathrm{HR1}^{\mathrm{MED} 24}$ of $\mathrm{MED}^{\mathrm{B}}$ remains associated with MED1 and MED15 ${ }^{\mathrm{C}}$ of the Shoulder, similar to that in $\mathrm{MED}^{\mathrm{E}}$. The MED16/24 core rotates downward by $\sim 50^{\circ}$ with the $\beta$-propeller of MED16 moving by up to $55 \AA$ and MED24 moving by up to $43 \AA$ (Fig. 4, B to D). Superimposition of the MED16/24 core shows the separation of MED23 and MED24 (Fig. 4E), indicting distinct modular arrangement within the Saddle module.

Cryo-EM classification of Mediator ${ }^{\mathrm{WT}}$ led to similar reconstructions of $\mathrm{MED}^{\mathrm{E}}$ and $\mathrm{MED}^{\mathrm{B}}$, indicating an effect independent of MED1-IDR (fig. S10, A and B). Additional states may remain in the data but have not been yet captured by refinement (figs. S2 and S10D). The deletion of MED25 does not affect Tail integrity or conformation (37), suggesting that the Tail-bending may not result from the potential dissociation of MED25. We suspected that the conformational heterogeneity of the Saddle may result from compositional heterogeneity of the purified Mediator, which may contain endogenous MED16 isoform-2 (built in the structural model 
of $\mathrm{MED}^{\mathrm{E}}$ ) and overexpressed MED16 isoform-1 (table S1). The $\mathrm{MED}^{\mathrm{B}}$ conformation was not observed in previous structural studies $(37,38,42)$ and differs from the Mediator heterogeneity in PIC ${ }^{\mathrm{TBP}}$-MED structure (42) (Fig. 4 and fig. S10). Thus, in addition to the known conformational heterogeneity of Mediator $(26-31,36-38,41,42,44)$, the presence of $\mathrm{MED}^{\mathrm{B}}$ conformation adds another layer of complexity in Mediator dynamics and the bent Tail may confer additional function(s) to accommodate transcription regulation.

\section{Distinct modular organization of Mediator in different species}

All previously reported mammalian Mediator structures reveal the $\mathrm{MED}^{\mathrm{E}}$ conformation $(37,38,42)$. Therefore, the structure of Mediator in the $\mathrm{MED}^{\mathrm{E}}$ conformation at nearatomic resolution is used in structural comparison below. The cryo-EM map of human Mediator (hMED) shows similar overall architecture and modular organization to the structure of mouse Mediator (mMED) at 4.0 $\AA$ resolution $(37,38)$, which lacks the structural model of MED26 and the models of MED1, MED10, MED19, and the N-terminal helices of MED14 were built by poly-alanine chain (fig. S11, A to $\mathrm{D}$, and supplementary text).

Structural comparison of hMED with yMed (27) and Chaetomium thermophilum Mediator (ctMed) (44) reveals considerable differences in modular organization (fig. S11, A, $\mathrm{E}, \mathrm{F}$, and G, and supplementary text). The structures of yMed and ctMed reveal more tilted Rod-Elbow (MED4/9MED1) and much smaller Tail in size due to the lack of metazoan-specific subunits. The yMed and ctMed lack about half of the THB and the majority of the Shoulder, resulting in obviously distinct organization of the Head-Shoulder. These differences are consistent with the metazoan-specific functions of Mediator in diversified transcription regulation in human cells.

\section{Structure determination of TFIID-based PIC-MED holo-complex}

Previous structural studies have focused on $\mathrm{PIC}^{\mathrm{TBP}}$ and $\mathrm{PIC}^{\mathrm{TBP}}$-Mediator complexes (39-42). However, PIC assembly and transcription initiation require the 14-subunit TFIID complex ( 1.3 MDa), which accounts for about half the mass of human holo-PIC ( 2.6 MDa) (Fig. 5, lower panel) and could not be functionally replaced by TBP $(6-9)$. To study how human Mediator is assembled into PIC, we reconstituted TFIID-based PIC complex as previously described (45) and assembled the PIC-Mediator complex (fig. S12), which consists of 76 polypeptides with molecular weight of $\sim 4.1$ MDa and represents the human PIC-Mediator holo-complex except for four residue-substitution in Pol II.

The cryo-EM 3D classification revealed four distinct conformational states (Fig. 5, figs. S12 to S15, movies S10 to
S13, and data S1). The first state was termed pre-hPIC-MED (Mediator-bound pre-assembled holo-PIC) and the last was termed hPIC-MED (Mediator-bound holo-PIC), in which the PIC resembles the previously described pre-hPIC and hPIC (45), respectively. The second and third states represent intermediate conformations and will not be discussed in detail below. In the four reconstructions, Mediator adopts generally similar overall fold (fig. S16A) but are distinctly placed relative to Pol II (fig. S15), indicating distinct PICMediator interactions and dynamics of PIC-Mediator assembly. The cryo-EM maps of pre-hPIC-MED and hPICMED were refined to 4.07 and $4.13 \AA$, respectively. Focused refinement largely improved the maps around Pol II (3.3 $\AA$ ), CTD-Mediator interface (3.7 $\AA$ ), TFIIH CAK (4.7 $\AA$ ), and other modules. Structural model was built according to the cryo-EM maps with the structures of PIC (45), MED ${ }^{\mathrm{E}}$, and $\mathrm{MED}^{\mathrm{B}}$ as templates (fig. S14 and data S1). The Mediator ${ }^{\mathrm{WT}}$ and Mediator ${ }^{\triangle \mathrm{MEDI}-\mathrm{IDR}}$ were used in complex assembly and structure determination, generating almost identical structures except that the PIC-Mediator ${ }^{\mathrm{MEDD}-\mathrm{IDR}}$ gave better cryoEM maps than that of PIC-Mediator ${ }^{\mathrm{WT}}$ (figs. S12 and S15, A to $\mathrm{E}$, and supplementary text). The pre-hPIC-MED reveals the best-resolved structure and thus is used in overall structure description.

\section{Overall structure of pre-hPIC-MED}

The pre-hPIC-MED structure reveals a globular fold and a bent Tail protrusion with approximate dimensions of $\sim 420 \times 370 \times 240 \AA^{3}$ (Fig. 5, figs. S17 and S18, and movie S10). Similar to that in pre-hPIC and hPIC (45), a central PIC core is formed by Pol II, TFIIF, TFIIB, TFIIA, and upstream promoter-bound TBP. The PIC core is surrounded by Mediator, the downstream promoter-bound TFIIH core, and the trilobular TFIID (termed IID-A, IID-B, IID-C submodules). IID$\mathrm{B}$ associates with the upstream promoter-bound TBP-TFIIA; and IID-C (TAF1/7 heterodimer and IID-C2) associates with the downstream promoter-bound TFIIH core. The TFIIH core adopts a horseshoe-shaped fold with XPB at one end bound to the promoter and XPD-MAT1 at the other end associated with TFIIE and Pol II stalk (RPB4 and RPB7). The TFIIH CAK is positioned on the exposed Head of Mediator and is tethered to the TFIIH core through MAT1.

Mediator is positioned above Pol II and makes multiple contacts with the PIC core, Pol II CTD, the TFIIH core, and CAK (Fig. 5, figs. S17 and S18, and movies S10 and S11). TFIIE, Pol II stalk, and the TFIIH core are sequentially arrayed below the Head of Mediator, generating intermodular contacts at this four-way junction. The MED18/20 heterodimer is positioned above Pol II dock. The Hook at one end of the Middle is sandwiched by the TFIIH core and CAK. The Rod-Elbow at the other end touches Pol II RPB8 and binds the Tail. The Tail, including the Shoulder and highly flexible Saddle, pro- 
trudes out of the main body and has no direct contact with PIC. Two well-ordered Pol II CTD segments wind through a Head-Middle sandwich with the longer one extending to CDK7, indicative of a Mediator-bridged CTD-CDK7 contact favorable for CTD phosphorylation.

\section{PIC-Mediator assembly leads to concerted reorganization of Mediator}

In pre-hPIC-MED, the Saddle of Mediator is notably bent toward Pol II, indicative of a conformation similar to that of $\mathrm{MED}^{\mathrm{B}}$, but not $\mathrm{MED}^{\mathrm{E}}$ (Fig. 6, A to $\mathrm{C}$, and fig. S16, B and $\mathrm{C}$ ). As in $\mathrm{MED}^{\mathrm{B}}$ (Fig. 4A), the HR1 ${ }^{\mathrm{MED} 23}$ and $\mathrm{HR}^{\mathrm{MED} 24}$ around the M-branch stably associate with the Head-Shoulder and Middle, respectively (Fig. 6, D and E). The rest of the Saddle are highly flexible and the $\mathrm{H}$-branch dissociates from the Head-Shoulder. The $\mathrm{MED}^{\mathrm{E}}$ conformation was not observed in PIC-Mediator structures (fig. S12), suggesting that PIC may prefer binding of the Mediator in the $\mathrm{MED}^{\mathrm{B}}$ conformation or facilitate transition from $\mathrm{MED}^{\mathrm{E}}$ to $\mathrm{MED}^{\mathrm{B}}$.

Superimposition of the Mediator in pre-hPIC-MED and hPIC-MED reveals generally similar fold except for slight rotation of the Middle due to the lack of contact between Rod-Elbow and Pol II in hPIC-MED (figs. S15, A and D, S16A, and S18C and movies S13 and S15). Structural comparison of Mediator in pre-hPIC-MED and MED in the apo form $\left(\mathrm{MED}^{\mathrm{E}}\right.$ in Fig. 6 and $\mathrm{MED}^{\mathrm{B}}$ in fig. S16) shows that the majority of the Head-Shoulder are well superimposed whereas the Middle and HB1-HB2 are distinctly organized, indicating considerable modular reorganization. Upon the assembly of PIC-Mediator complex, the HB1-HB2 of the Head tilts (Head-tilting) toward the Middle and the Hook and Knob of the Middle move downward (Middle-down), generating the Head-Middle sandwich. The two CTD segments serve as a double-sided sticker to bridge the otherwise separated Head and Middle. The Head-tilting involves a rotation of the $\mathrm{HB} 1-\mathrm{HB} 2$ by $\sim 15^{\circ}$ and a movement of $\alpha \beta^{\mathrm{MED} 6}$ by as far as $27 \AA$, creating complementary surfaces of Mediator and Pol II stalk (fig. S18A). The HB1-HB2 in the apo form would clash with Pol II stalk and the TFIIH core if not tilted (Fig. 6F). The Middle-down involves downward movements of the Hook ( $\sim 50 \AA)$ and Knob $(\sim 20 \AA)$ and slightly upward movements of the Rod and Elbow ( $\sim 5)$, generating a rotation of the Middle by $\sim 20^{\circ}$.

\section{A two-lever model of PIC-induced reorganization of Mediator}

As exemplified by the transition from $\mathrm{MED}^{\mathrm{B}}$ to PIC-MED, the concerted movements of Mediator are coordinated by two molecular levers that are hinged by the flexible RodElbow tether (Fig. 6G, figs. S9A and S18C, and movie S14). Binding of PIC results in downward movements of the Hook and Knob and upward movements of the Rod (MED4/9) and Elbow (MED1) through the lever-I, which is formed by the Hook and Rod with RM1 ${ }^{\mathrm{MED14}}$ serving as a moveable fulcrum. The movement of lever-I is coordinated with slight movement of the lever-II, which is formed by MED1 and $\mathrm{HR1}^{\mathrm{MED} 24}$ with MED15 ${ }^{\mathrm{C}}$ serving as a fixed fulcrum. The slight rotation of $\mathrm{HR}^{\mathrm{MED} 24}$ may facilitate the Saddle dynamics in PIC-Mediator. Consistent with this model, the deletion of MED1 in mouse Mediator leads to a similar Middle-down movement (37), suggesting that the Tail-associated MED1 restrains the Middle in an upwardly tilted conformation. Despite no direct contact with Pol II, the Tail is the major target of TFs $(21,55)$ and involves regulation of transcription initiation (56-60) (fig. S19), suggesting a potential role of the Tail dynamics in modulating interactions between TFs and Pol II for transcription regulation.

Structural studies of yMed $(27,28)$ and ctMed (44) indicated that Pol II induces conformational changes of Mediator and bring the Head and Middle into contact (fig. S16, D and E), suggesting similar CTD-Mediator interactions, albeit the lack of structurally defined density of CTD. Pol II-yMed assembly results in slight Tail-bending toward Pol II (27) (fig. S16E). Thus, despite distinct Tail organization in different species (fig. S9), binding of Pol II and PIC may induce conserved movements of the Head and Middle modules.

\section{Dynamic interactions between Mediator and PIC}

The pre-hPIC-MED and hPIC-MED structures reveal distinct patterns of interactions between Mediator and PIC, suggesting dynamics of PIC-Mediator organization dependent on PIC conformation (Fig. 7A, fig. S18, and movie S15).

Besides the CTD-Mediator interactions (discussed below), four PIC-Mediator contacts were observed in pre-hPICMED (Fig. 7, B to E, left panels, and fig. S18): (I) The HB1HB2 of the Head sits on top of RPB4 of Pol II stalk with the helix hairpin of MED8 packing against helices $\alpha 3$ and $\alpha 6$ of RPB4, generating a Head-stalk contact (Fig. 7B). (II) MED18 and MED20 are placed above Pol II dock and RPB3/11 heterodimer, respectively, generating complementary binding surfaces (Fig. 7C). The N-terminal segment of MED18 anchors into Pol II through packing against Pol II dock above the active site. (III) MED4/9 helix bundle and MED1 at the Rod-Elbow tether sit on top of Pol II RPB8 (Fig. 7D). (IV) The Hook is sandwiched by the TFIIH CAK and XPB of the TFIIH core with the end touching XPD (Fig. 7E). The above four contacts are separated on four corners of the Head and Middle and position Mediator above PIC (Fig. 7A, right panel), consistent with the well-resolved Mediator structure. The yeast PIC ${ }^{\mathrm{TBP}}-\mathrm{MED}$ and $\mathrm{PIC}^{\mathrm{TBP}}$-cMED structures $(40,41)$ show similar PIC-Mediator binding patterns except for the lack of Hook-XPB contact at the interface-IV (fig. S20, movie S16, and supplementary text).

In contrast to the four contacts in pre-hPIC-MED, the 
hPIC-MED structure shows the lack of intermodular contacts at the interface-II/III but more extensive Head-stalk contacts at the interface-I (Fig. 7, B to E, right panels, and fig. S18). From the Tip domain to the OB domain, Pol II stalk makes contacts with the RM1 and RM2 of MED14, the helix bundle HB1, and a MED8 linker bridging the HB2 and MED18/20 (Fig. 7B). Around the interface-IV, the Hook contacts XPB, but does not touch XPD (Fig. 7E). The interactions between Mediator and PIC are generally similar to that in human PIC ${ }^{\mathrm{TBP}}$-MED structure (42) except for the differences in the interactions around the interface-IV and the CTD-binding site (fig. S21, movies S17 and S18, supplementary text).

\section{Dynamics of PIC-Mediator assembly}

Similar to that in PIC $^{\mathrm{TBP}}$ (39) and PIC ${ }^{\mathrm{TBP}}-\mathrm{MED}(40-42)$ structures, the promoter of hPIC-MED is positioned above Pol II cleft and is ready for promoter melting and transcription initiation, indicating that hPIC-MED represents a fullyassembled state whereas the pre-hPIC-MED is a preassembled state (Fig. 7, figs. S15, S20, and S21, and movies S12 and S15). Structural comparison suggests a transition from pre-hPIC-MED to hPIC-MED, during which the movements of the promoter, TFIID, and promoter-bound TFIIH core relative to Pol II lead to coordinated movements of TFIIE, Pol II stalk, and Mediator through intermodular associations around the four-way junction.

In pre-hPIC-MED, TAF1/7 (IID-C1) of TFIID remains associated with the downstream promoter, relative to transcription start site (TSS) (Fig. 7, A and F, figs. S17 and S18, and movies S10, S11, and S15). The high-mobility group of TAF1 (HMG ${ }^{\mathrm{TAF}}$ ) is placed between the promoter and Pol II jaw and prevents further promoter deposition to Pol II. During the transition to hPIC-MED, TAF1/7 and the closely associated $\mathrm{HMG}^{\mathrm{TAF}}$ dissociate from the promoter, permitting promoter deposition to Pol II. TFIID supports the promoterbound XPB to move toward and contact Pol II jaw (45), accompanied by the movement of the TFIIH core. The movement is transferred to the TFIIH core-associated TFIIE and Pol II stalk and results in a rotation of stalk-bound Mediator by $\sim 20^{\circ}$ relative to Pol II. The movement transduction from TFIID-promoter to TFIIE-TFIIH and then to Pol II stalk and Mediator suggest that the architecture of PIC modulates the placement of Mediator but not vice versa (Fig. 7A, fig. S15, and movie S15). TFIID may play a regulatory role in the well-accepted complexity and dynamics of PIC-Mediator on core promoters (61-63).

\section{TFIID and Mediator orchestrate PIC-Mediator organization and TFIIH stabilization}

In pre-hPIC-MED and hPIC-MED, Mediator indirectly stabilizes the TFIIH core at the four-way junction and directly contacts the TFIIH CAK, XPB, and the helix hairpin of MAT1 (Fig. 7, A and E, and figs. S18D and S20A). Thus, although the placement of Mediator relative to Pol II is regulated by PIC conformation, the network interactions of Mediator and TFIIH may facilitate incorporation of TFIIH into PIC and/or stabilize TFIIH in PIC-Mediator, consistent with the function of Mediator in supporting PIC assembly and stimulating basal transcription activity (64-66) (fig. S1E).

TFIID binds the p52/p8 heterodimer of the TFIIH core and supports XPB to be positioned upward to contact Pol II jaw and the Hook of Mediator (Fig. 5A and Fig. 7, E and F). It has been reported that p52/p8 stimulates XPB ATPase activity $(67,68)$. TFIID and Mediator may together sandwich and stabilize the promoter-bound $\mathrm{p} 52 / \mathrm{p} 8 / \mathrm{XPB}$ subcomplex in PIC-Mediator and facilitate XPB-mediated promoter melting and DNA translocation toward Pol II for transcription initiation.

The network interactions around XPB have escaped previous studies in TBP-based system (40-42), in which XPB appears to be less stabilized due to the lack of contacts with Pol II jaw, TFIID, and the Hook (Fig. 7E, figs. S20 and S21, and movies S16 and S17). The conformational differences may reflect the difference in complex composition (TFIID versus TBP), underscoring the critical role of TFIID in orchestrating PIC-Mediator organization and TFIIH stabilization.

\section{Mediator positions the TFIIH CAK}

The TFIIH core and the main body of the CAK have no direct contact and are tethered by MAT1, in which the Nterminal domain $\left(\mathrm{MAT1}^{\mathrm{N}}\right)$ associates with XPD, TFIIE, and Pol II stalk, a helix hairpin bridges XPD and XPB, the following linker winds over the $\alpha \beta^{\mathrm{MED} 6}$ of the HB1, and the Cterminal region $\left(\mathrm{MAT1}^{\mathrm{C}}\right.$ ) is incorporated into the CAK (Fig. 8, A to D, fig. S22, and movie S19). The CAK is positioned on the exposed HB1 of the Head through the contact between $\alpha \beta^{\mathrm{MED} 6}$ and the C-terminal lobe (C-lobe) of CDK7. The relatively small CAK-Mediator interface is consistent with the observed flexibility of the CAK.

The CAK placement in pre-hPIC-MED and hPIC-MED structures agrees with the observations in yeast PIC ${ }^{\mathrm{TBP}}$-MED structure (40) (fig. S20) and human PIC ${ }^{\text {TBP }}$-MED structure (42) (fig. S21), indicating evolutionarily conserved positioning of the CAK in PIC-Mediator complex $(40,42)$. Intriguingly, in Mediator-free hPIC (45), the CAK is positioned near Pol II foot and residue P1487, the last modeled RBP1 residue preceding the linker and CTD, indicating a position favorable of CTD phosphorylation. The CAK in this position would clash with Mediator in hPIC-MED (fig. S15H). The two mutually exclusive CAK-binding sites in PIC and PICMediator may ensure efficient CTD phosphorylation in the presence (high transcription activity) and absence (low 
transcription activity) of Mediator in different transcription contexts $(51,69)$.

\section{Two CTD segments are sandwiched by the Head and Middle in PIC-Mediator}

Two segments of CTD heptapeptide repeats $\left(\mathrm{Y}^{1} \mathrm{~S}^{2} \mathrm{P}^{3} \mathrm{~T}^{4} \mathrm{~S}^{5} \mathrm{P}^{6} \mathrm{~S}^{7}\right)$ are sandwiched by the Knob of the Middle and HB1 of the Head (Fig. 8, A and E to K, fig. S22, and movies S19 and $\mathrm{S} 20$ ). The Knob and $\mathrm{HB1}$ have no direct contact but are bridged by the two CTD segments, in which the $\mathrm{Y}^{1}$ residues serve as arrayed anchors in guiding the CTD to wind through the Head-Middle sandwich. Although the $\mathrm{Y}^{1}, \mathrm{P}^{3}$, and $\mathrm{P}^{6}$ residues fit well into the cryo-EM map, the repetitive CTD is almost symmetric $\left(\mathrm{Y}^{1} \mathrm{~S}^{2} \mathrm{P}^{3} \mathrm{~T}^{4} \mathrm{~S}^{5} \mathrm{P}^{6} \mathrm{~S}^{7} \mathrm{Y}^{1} \mathrm{~S}^{2} \mathrm{P}^{3} \mathrm{~T}^{4} \mathrm{~S}^{5} \mathrm{P}^{6} \mathrm{~S}^{7} \mathrm{Y}^{1}\right)$ and the CTD direction could not be unambiguously determined solely from the cryo-EM map. The directions of the two CTD segments were proposed according to their topological placement relative to Pol II and the structure of CTD in complex with the Head of yMed (70).

Within the Head-Middle sandwich, the U-shaped shorter segment (CTD-S, 10 well-ordered residues) packs on a shallow groove of the Knob and the two $\mathrm{Y}^{1}$ residues make hydrophobic contact with residue $\mathrm{P} 83^{\mathrm{MED} 8}$ of $\mathrm{HB1}$ (Fig. $8 \mathrm{~F}$, right panel). The longer segment (CTD-L, 25 well-ordered residues) consists of 3 complete repeats (CTD-LR2 to CTDLR4) with one end positioned $\sim 70 \AA$ away from the last modeled RPB1 residue (P1487) in the PIC core (Fig. 8, F to J, and fig. S22). Residue $\mathrm{Y}^{1}$ of CTD-LR2 contacts F96 ${ }^{\mathrm{MED} 31}$ and C93 ${ }^{\text {MED31 }}$ of the Knob (Fig. 8G). Residue $\mathrm{Y}^{1}$ of CTD-LR3 generates a hydrogen bond with R118 ${ }^{\mathrm{MED} 6}$ and hydrophobic contact with $\mathrm{V} 111^{\mathrm{MED17}}$ of the HB1 (Fig. $8 \mathrm{H}$ ). Residue $\mathrm{Y}^{1}$ of CTDLR4 inserts into a hydrophobic pocket formed by $\mathrm{Y}_{106}{ }^{\mathrm{MED} 6}$ and P106 ${ }^{\mathrm{MED} 8}$ of the HB1 (Fig. 8I). The CTD-Mediator interactions are further supported by other contacts, as exemplified by the N-terminal tail of MED7 spanning the CTD-LR4 (Fig. 8, E and F). The locally refined cryo-EM map of PIC$\mathrm{MED}^{\mathrm{WT}}$ reveals similar Head-Middle sandwich and stabilization of two CTD segments, indicating that the MED1-IDR does not affect CTD-Mediator interaction (fig. S22G). The CTD-binding pattern differs from that observed in the crystal structure of CTD-Head of yMed (70), possibly due to the lack of stabilization by the Knob in the crystal structure (fig. S22, E and F).

\section{Mediator brings Pol II CTD into contact with CDK7}

Cryo-EM map shows a putative CTD-L extension (CTD-LR5 to CTD-LR6), which protrudes out of the Head-Middle sandwich and packs against the C-lobe of CDK7 with the terminus positioned $\sim 15 \AA$ away from the CDK7 active site (Fig. 8D, fig. S22C, and movie S19). The placement of CTD agrees with the observed CTD-CDK7 contact in PIC ${ }^{\mathrm{TBP}}-\mathrm{MED}$ (42) and is consistent with the XL-MS, which shows cross- links of CTD residues with subunits of the CAK (CDK7/CycH), Knob (MED31), and Hook (MED10/19) (fig. S4D and data S3). This architectural arrangement indicates that Mediator brings Pol II CTD and CDK7 into contact, in line with the function of Mediator in stimulating CDK7mediated CTD phosphorylation (15).

\section{A model of Mediator-stimulated CTD phosphorylation}

Pol II CTD consists of 52 heptapeptide repeats in humans and 26 repeats in yeast. The CTD-Mediator binding pattern may reveal how the long repetitive CTD undergoes phosphorylation in PIC-Mediator complex. The CTD-L and CTD$\mathrm{S}$ are positioned on top and bottom of the Head-Middle sandwich, respectively (Fig. 8E), and the two nonoverlapping sites separate the CTD into exposed (eCTD) and cradled (cCTD) portions (Fig. 8, J and K). The Head-Middle sandwich may provide stable CTD-anchoring sites and facilitate efficient access of the anchored eCTD to CDK7 for phosphorylation.

The Knob and HB1 of the Head-Middle sandwich have no direct interaction (Fig. 8E) and undergo considerable reorganization during assembly of PIC-Mediator and slight reorganization during transition from pre-hPIC-MED to hPIC-MED (Fig. 6, fig. S16, and movies S14 and S15). The dynamics of the Head-Middle sandwich suggests a CTDgating mechanism, by which one CTD segment remains sandwiched and maintains CTD-Mediator association whereas the more separated sandwich at the other side allows the originally sandwiched/cradled CTD to pass through the gate for phosphorylation (Fig. 8K). The putative dynamics of the Head-Middle sandwich might result from relative movement between the Head and Middle, which has been observed in the binding of Mediator to Pol II (27, 28, 44), PIC (40-42), and transcription factors $(30,71)$.

Previous studies showed that CTD is necessary and sufficient for binding of Mediator and CTD phosphorylation leads to dissociation of Mediator from Pol II (41, 63, 72-74), suggesting that the acidic and bulky phosphate groups of the phosphorylated $S^{5}$ residues may generate steric clash and/or charge repulsion with Mediator and disrupt the binding of CTD to Head-Middle sandwich (Fig. 8F). Thus, Mediator may not only facilitate CTD-CDK7 interaction for reaction but also ensure persistent CTD phosphorylation by sending unphosphorylated CTD to CDK7. Nearly complete CTD phosphorylation leads to dissociation of Mediator from PIC and allows for promoter escape of Pol II and productive transcription $(51,63)$.

The Hook associates with the Knob at one side of the Head-Middle sandwich (Fig. 5A, right panel) and bridges the TFIIH CAK and XPB (Fig. 7E). At the other side of the Head-Middle sandwich, the HB1-HB2 binds Pol II stalk and the TFIIH CAK (Fig. 8A). The physical association among 
$\mathrm{XPB}, \mathrm{CDK} 7$, and Head-Middle sandwich suggests a functional coordination of CDK7-mediated CTD phosphorylation and XPB-mediated promoter melting, the two critical reactions required for transcription initiation.

The yeast PIC ${ }^{\mathrm{TBP}}$-MED/cMED structures show HeadMiddle sandwich, but no CTD model was built due to the limitation of the cryo-EM maps $(40,41)$ (fig. S20). The human PIC ${ }^{\mathrm{TBP}}$-MED structure (42) shows conformational heterogeneity of Mediator, relatively open Head-Middle sandwich, and the lack of binding to the CTD-S segment (fig. S21, movie S17, and supplementary text), providing an example of the CTD-gating model. Compared to that in preh/hPIC-MED structures, the Hook is incompletely positioned downward (incomplete Middle-down) in PIC ${ }^{\mathrm{TBP}}$-MED and leads to an incomplete Head-Middle sandwich, which partially stabilizes the CTD-L (14 traced residues in PIC ${ }^{\text {TBP }}$ MED versus 25 well-ordered residues in pre-h/hPIC-MED) and does not stabilize the CTD-S (invisible in PIC ${ }^{\text {TBP }}$-MED versus 10 well-ordered residues in pre-h/hPIC-MED). The difference in CTD-Mediator interaction in PIC ${ }^{\mathrm{TBP}}$-MED and TFIID-based PIC-MED highlights the critical role of TFIID in assembly of a functional PIC-Mediator complex.

\section{Materials and methods Construction of Mediator expression plasmids}

Full length open reading frames (ORFs) of 26 human Mediator subunits and MED1 ${ }^{\triangle I D R}$ (1-527aa) were sub-cloned into a modified pCAG vector (75) with or without a protein A (ProA) tag at the $\mathrm{N}$ terminus. Two or three ORFs of Mediator subunits were inserted into one expression vector to improve protein expression level and homogeneity. Iterative optimization generated eight integrated plasmids as follows, MED17-MED14, MED6-MED20 (MED20 tagged), MED18MED8, MED4-MED9-MED21, MED22-MED30-MED11, MED7-MED31-MED26 (MED26 tagged), MED10-MED19, MED27-MED29-MED28. Each of the ORFs was sub-cloned into a multiple cloning site following individual promoter, generating a multicistronic expression plasmid as previously reported (75). The rest six untagged large subunits (MED1, MED15, MED16, MED23, MED24, MED25) were individually inserted into the pCAG vector and co-transfected with the eight multicistronic expression plasmids.

Similar approaches were performed for Mediator ${ }^{\mathrm{WT}}$ (containing full length MED1) and Mediator ${ }^{\mathrm{MEDI}-\mathrm{IDR}}$ in protein expression, purification, cryo-EM grid preparation, data collection, image processing, and structure determination. The cryo-EM maps of Mediator ${ }^{\triangle M E D 1-I D R}$ and PIC-MED ${ }^{\triangle M E D 1-I D R}$ were used for building of the structural models.

\section{Purification of the recombinant human Mediator complex}

All human Mediator subunits (14 plasmids) were co- transfected into suspension Expi293F cells using PEI (25000 MW, Polysciences) when the cells reach a density of $2 \times 10^{6} / \mathrm{ml}$. A process of repeated testing with various combinations and titrations of these tagged or non-tagged plasmids led to a near stoichiometric recombinant complex. The purified complexes were subjected to $4-12 \%$ SurePAGE BisTris gel (GenScript Biotech) and stained with Coomassie Blue R-250 or silver staining.

After culturing at $37^{\circ} \mathrm{C}$ for 66 hours, cells were harvested by centrifugation at $4000 \mathrm{rpm}$ for $15 \mathrm{~min}$ (Cence centrifuge L720L-3, rotor \#3). Cells were lysed using buffer containing $25 \mathrm{mM}$ HEPES-NaOH, pH 8.0, $300 \mathrm{mM} \mathrm{NaCl}$, $0.2 \%$ CHAPS, $10 \%$ glycerol, $5 \mathrm{mM} \mathrm{MgCl}_{2}, 5 \mathrm{mM}$ ATP, $0.5 \mathrm{mM}$ EDTA, $2 \mathrm{mM}$ DTT and $0.1 \mathrm{mM}$ PMSF supplemented with protease inhibitor cocktail. Cell debris was removed through centrifugation at 4,000 rpm for $10 \mathrm{~min}$. Then the crude extracts were further clarified by centrifugation at 16,000 rpm for 30 min with JLA-16.250 rotor (Beckman Coulter). The supernatant was incubated with IgG-agarose resin (Smart Lifesciences) at $4^{\circ} \mathrm{C}$ for 2 hours and washed with binding buffer including $0.05 \%$ CHAPS and no protease inhibitors. Immunoprecipitated Mediator complex was subjected to oncolumn digestion overnight. The eluate fractions were further purified using Mono Q (5/50 GL, GE healthcare) anionexchange chromatography. Fractions containing stoichiometric Mediator complex were pooled, concentrated to $\sim 1 \mu \mathrm{g} / \mu \mathrm{l}$ and flash-frozen in liquid nitrogen or used for subsequent analysis. For each purification step, proteins were monitored with purity and stoichiometry by SDS-PAGE. Typically, for each preparation, $\sim 2 \mathrm{mg}$ of purified Mediator could be obtained from $8 \mathrm{~L}$ cells.

\section{Protein expression and purification of PIC components}

TFIID, TFIIA, TFIIB, TFIIF, TFIIE and TFIIH were expressed and purified as previously described (45). Pol II was isolated from $S$. scrofa thymus and purified following the reported protocol (76). Four residue substitutions (G882S of RBP2, T75I of RPB3, S140N of RPB3, and S126T of RPB6) exist between $S$. scrofa and $H$. sapiens Pol II.

\section{Promoter DNA preparation}

To assemble the PIC-Mediator complex, the super core promoter $\left(\mathrm{SCP}\right.$ or $\left.\mathrm{CP}^{\mathrm{SCP}}\right)(39,77)$ was used. The $\mathrm{CP}^{\mathrm{SCP}}$ sequence was based on the reported SCP promoter and introduced a BREu element into the upstream of TATA box as previously reported (39, 77) (template $5^{\prime}$ GATATCCATGGTCCGTAGGCACGTCTGCTCGGCTCGAGTGT TCGATCGCGACTGAGGACGAACGCGCCCCCACCCCCTTTTA TAGGCGCCCTTCGATATC-3'; non-template $5^{\prime}$ ' GATATCGAAGGGCGCCTATAAAAGGGGGTGGGGGCGCGTT CGTCCTCAGTCGCGATCGAACACTCGAGCCGAGCAGACGT 
GCCTACGGACCATGGATATC-3' ') (the SCP sequence is underscored).

The six repeats of $\mathrm{CP}^{\mathrm{SCP}}$ sequences were synthesized and sub-cloned into pMV vector to yield the pMV-6X-SCP plasmid. The DNA fragments for PIC-Mediator complex assembly were isolated from the pMV-6X-SCP plasmid by digestion with EcoRV (New England BioLabs), followed by ion-exchange chromatography (Mono Q, 5/50 GL, GE Healthcare) and isopropanol precipitation. The purified DNA pellet was dissolved in water.

\section{In vitro transcription assay}

In vitro transcription assay was performed according to previous studies $(35,78)$. Briefly, 1.3 pmol of SCP promoter DNA was combined with 1.5 pmol of TFIID, 3 pmol of TFIIA, 3 pmol of TFIIB, 3 pmol of TFIIF, 3 pmol of TFIIE, 1.5 pmol of TFIIH, 2 pmol of Pol II and increasing amount of the Mediator ${ }^{\mathrm{WT}}$ and Mediator ${ }^{\triangle \mathrm{MEDI}-\mathrm{IDR}}(0 \mathrm{pmol}, 2 \mathrm{pmol}$ and $4.5 \mathrm{pmol}$ ) in a volume of $10 \mu \mathrm{l}$ containing $30 \mathrm{mM}$ HEPES pH $7.9,100 \mathrm{mM} \mathrm{KCl}, 6 \mathrm{mM} \mathrm{MgCl} 2,2 \mathrm{mM}$ DTT, $5 \%$ (v/v) glycerol for $30 \mathrm{~min}$ at $25^{\circ} \mathrm{C}$. Reactions were initiated by the addition an equal volume of buffer containing $24 \mathrm{mM}$ HEPES-KOH pH 8.0, $120 \mathrm{mM} \mathrm{KCl}, 10 \mathrm{mM} \mathrm{MgCl}_{2}, 1.2 \mathrm{mM}$ DTT, 24\% (v/v) glycerol, $100 \mu \mathrm{g} / \mathrm{ml} \mathrm{BSA,} 200 \mu \mathrm{M}$ GTP, $200 \mu \mathrm{M} \mathrm{CTP}, 200 \mu \mathrm{M}$ ATP, $200 \mu \mathrm{M}$ UTP, and $99 \mathrm{nM}\left[\alpha^{-32} \mathrm{P}\right]$ UTP. The reactions were incubated at $25^{\circ} \mathrm{C}$ for $30 \mathrm{~min}$ and then were subjected to urea polyacrylamide gels and autoradiography.

\section{Cryo-EM sample preparation of Mediator}

To further purify the Mediator complex (Mediator ${ }^{\mathrm{WT}}$ or Mediator $^{\triangle \mathrm{MEDI}-\mathrm{IDR}}$ ) for EM studies, $\sim 100 \mathrm{pmol}$ of protein aliquot was subjected to $15-35 \%$ glycerol gradient ultracentrifugation with swinging bucket rotor SW41Ti (Beckman) at $38000 \mathrm{rpm}$ at $4^{\circ} \mathrm{C}$ for 16 hours. The purified protein complex was verified by SDS-PAGE and negative stain electron microscopy (EM). Gradient fixation (GraFix) (79) was performed in the following cryo-EM sample preparation, in which the chemical fixation reagent glutaraldehyde (Sigma) was added in the denser glycerol solution. Varying concentrations of glutaraldehyde were tested to acquire more intact protein complexes and less aggregates $(0.01 \%$ in this case). Fractions containing monodispersed complexes were pooled and concentrated. The final concentration of glycerol in the GraFix-prepared samples was decreased to below 1\% by dialysis or buffer exchange with a spin column.

For negative stain EM grids preparation, $5 \mu \mathrm{l}$ of Mediator complex sample were applied onto glow-discharged copper grids supported by a continuous thin layer of carbon film for $60 \mathrm{~s}$ before negatively stained by $2 \%(\mathrm{w} / \mathrm{v})$ uranyl formate solution at room temperature. The grids were prepared in the $\mathrm{Ar} / \mathrm{O}_{2}$ mixture for $15 \mathrm{~s}$ using a Gatan 950 Solarus plasma cleaning system with a power of $15 \mathrm{~W}$. The negatively stained grids were loaded onto a Thermo Fisher Scientific Talos L120C microscope equipped with a Ceta CCD camera and operating at $120 \mathrm{kV}$ at a nominal magnification of $92,000 \mathrm{x}$, corresponding to a pixel size of $1.58 \AA$ on the specimen.

For cryo-EM grids preparation, $3 \mu \mathrm{l}$ of the sample at a concentration of $\sim 0.4 \mathrm{mg} / \mathrm{ml}$ Mediator complex were applied to freshly glow-discharged Quantifoil R1.2/1.3 Au holey carbon grids. After incubation of $5 \mathrm{~s}$ at a temperature of $4{ }^{\circ} \mathrm{C}$ and a humidity of $100 \%$, the grids were blotted for 1-1.5 $\mathrm{s}$ in Vitrobot Mark IV (Thermo Fisher Scientific) and plungefrozen in liquid ethane and stored at liquid nitrogen temperature. The grids were prepared in the $\mathrm{H}_{2} / \mathrm{O}_{2}$ mixture for 45 s using a Gatan 950 Solarus plasma cleaning system with a power of $5 \mathrm{~W}$. The $\varnothing 55 / 20 \mathrm{~mm}$ blotting paper is made by TED PELLA used for plunge freezing.

\section{Cryo-EM sample preparation of PIC-Mediator}

Samples of PIC-MED ${ }^{\mathrm{WT}}$ and PIC-MED ${ }^{\triangle \mathrm{MEDI}-\mathrm{IDR}}$ were prepared in an almost identical approach. The purified TFIID complex was incubated with TFIIA, TFIIB and promoter DNA $\left(\mathrm{CP}^{\mathrm{SCP}}\right)$ in a molar ratio of $1: 10: 10: 2$ at $4^{\circ} \mathrm{C}$ for $3 \mathrm{~h}$. The mixture of CP ${ }^{\mathrm{SCP}}$, TFIID, TFIIA, TFIIB was added on top of a 4 $\mathrm{mL} 10 \%-50 \%(\mathrm{w} / \mathrm{v})$ glycerol gradient in buffer containing 30 $\mathrm{mM}$ HEPES-KOH pH 7.9, $50 \mathrm{mM} \mathrm{KCl}, 6 \mathrm{mM} \mathrm{MgCl}_{2}, 2 \mathrm{mM}$ DTT and centrifuged at $36000 \mathrm{rpm} 4^{\circ} \mathrm{C}$ for 14 hours using an SW60 Ti rotor (Beckman Coulter). The fractions, 200 $\mu \mathrm{l}$ each, were collected manually from the top of the gradient and analyzed by an SDS-PAGE gel followed by Coomassie blue staining. Peak fractions containing CP ${ }^{\text {SCP }}$-TFIID-TFIIATFIIB complex were pooled, concentrated and incubated with the purified Pol II, TFIIF and Mediator in a molar ratio of 1:1.5:3:1.1 at $4^{\circ} \mathrm{C}$ for 2 hours. Subsequently, a fourfold molar excess of TFIIE and twofold molar excess of TFIIH (rela-

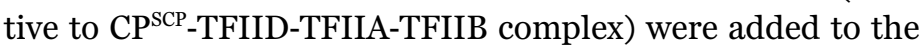
pre-incubated mixture of CP ${ }^{\text {SCP }}$-TFIID-TFIIA-TFIIB, Mediator, Pol II, and TFIIF, followed by additional incubation for 2 hours. The mixture was then subjected to GraFix (80). The glycerol gradient was prepared using light buffer containing $15 \%$ (v/v) glycerol, $30 \mathrm{mM}$ HEPES-KOH pH 7.9, $100 \mathrm{mM} \mathrm{KCl}$, $6 \mathrm{mM} \mathrm{MgCl} 2,2 \mathrm{mM}$ DTT, and heavy buffer containing 35\% (v/v) glycerol, $30 \mathrm{mM}$ HEPES-KOH pH 7.9, $100 \mathrm{mM} \mathrm{KCl,} 6$ $\mathrm{mM} \mathrm{MgCl}_{2}, 2 \mathrm{mM}$ DTT, and $0.015 \%$ glutaraldehyde. The samples were centrifuged at $33,000 \mathrm{rpm}$ at $4^{\circ} \mathrm{C}$ for 14 hours using an SW41 Ti rotor (Beckman Coulter). Subsequently, fractions containing cross-linked complexes were quenched with $50 \mathrm{mM}$ Tris $\mathrm{pH} 7.4\left(25^{\circ} \mathrm{C}\right)$. The homogeneity of peak fractions was assessed by negative stain EM. Fractions of interest were pooled, concentrated, followed by buffer exchange into a buffer containing $30 \mathrm{mM}$ HEPES-KOH pH 7.9, $100 \mathrm{mM} \mathrm{KCl}, 6 \mathrm{mM} \mathrm{MgCl} 2,2 \mathrm{mM}$ DTT, and $0.8 \%$ (v/v) glycerol. 
Negative stain EM grids preparation was performed as described above. For cryo-EM grids preparation, $3.5 \mu \mathrm{l}$ of the sample at a concentration of $0.5 \mathrm{mg} / \mathrm{ml}$ PIC-Mediator complex were applied to freshly glow-discharged Quantifoil $\mathrm{R} 2 / 2$ gold holey carbon grids. At a temperature of $4^{\circ} \mathrm{C}$ and under a humidity of $100 \%$, the grids were blotted for $0.5 \mathrm{~s}$ using a FEI Vitrobot Mark IV (FEI) and plunge frozen in liquid ethane cooled by liquid nitrogen. The grids were prepared in the $\mathrm{H}_{2} / \mathrm{O}_{2}$ mixture for $20 \mathrm{~s}$ using a Gatan 950 Solarus plasma cleaning system with a power of $5 \mathrm{~W}$.

\section{Data collection}

The cryo-EM grids of Mediator ${ }^{\mathrm{WT}}$ were loaded onto a Thermo Fisher Scientific Titan Krios G3i transmission electron microscope operated at $300 \mathrm{kV}$ for data collection. All the cryo-EM images were automatically recorded by K3 Bioquantum direct electron detector in the super-resolution counting mode using Serial-EM software (81) with a nominal magnification of $22,500 \mathrm{x}$, which yielded a superresolution pixel size of $0.53 \AA$ on the image plane, and with a defocus ranging from -1.0 to $-3.5 \mu \mathrm{m}$. Each micrograph stack was dose-fractionated into 40 frames with a total electron dose of $\sim 50 \mathrm{e}-/ \AA^{2}$ and a total exposure time of $3.12 \mathrm{~s}$. 7468 micrographs from a total of 8109 micrographs were selected for further processing.

The cryo-EM grids of Mediator ${ }^{\triangle \mathrm{MEDI}-\mathrm{IDR}}$ were loaded onto a Thermo Fisher Scientific Titan Krios G3 transmission electron microscope equipped with a Gatan GIF Quantum energy filter (slit width $20 \mathrm{eV}$ ) and operated at $300 \mathrm{kV}$ for data collection. All the cryo-EM images were automatically recorded by a post-GIF Gatan K2 Summit direct electron detector in the super-resolution counting mode using Serial-EM software (81) with a nominal magnification of $130,000 \mathrm{x}$, which yielded a super-resolution pixel size of $0.527 \AA$ on the image plane, and with a defocus ranging from -1.0 to -3.5 $\mu \mathrm{m}$. Each micrograph stack was dose-fractionated into 32 frames with a total electron dose of $\sim 50 \mathrm{e}^{-} / \AA^{2}$ and a total exposure time of $7.04 \mathrm{~s}$. 16,423 micrographs from a total of 17,763 micrographs were selected for further processing.

The cryo-EM grids of PIC-MED ${ }^{\mathrm{WT}}$ were loaded onto a Thermo Fisher Scientific Titan Krios G3i transmission electron microscope equipped with a Gatan GIF Quantum energy filter (slit width $20 \mathrm{eV}$ ) and operated at $300 \mathrm{kV}$ for data collection. All the cryo-EM images were automatically recorded by a post-GIF Gatan K2 Summit direct electron detector in the super-resolution counting mode using Serial-EM software (81) with a nominal magnification of 105,000 x, which yielded a super-resolution pixel size of $0.69 \AA$ on the image plane, and with a defocus ranging from -1.0 to -3.5 $\mu \mathrm{m}$. Each micrograph stack was dose-fractionated into 32 frames with a total electron dose of $\sim 50 \mathrm{e}^{-} / \AA^{2}$ and a total exposure time of 9.6 s. 8341 micrographs from a total of
9033 micrographs were selected for further processing.

The cryo-EM grids of PIC-MED ${ }^{\triangle M E D 1-I D R}$ were loaded onto a Titan Krios transmission electron microscope equipped with a Gatan K2 Summit direct electron detector and operated at $300 \mathrm{kV}$ for data collection. 11,583 micrographs were collected for further processing. Each micrograph stack was dose-fractionated to 32 frames with a total electron dose of $\sim 50 \mathrm{e}^{\mathrm{e}} / \AA^{2}$ and a total exposure time of $6.94 \mathrm{~s}$. The calibrated physical pixel size and the super-resolution pixel size are $1.054 \AA$ and $0.527 \AA$, respectively.

\section{Image processing}

For cryo-EM data, drift and beam-induced motion correction were applied on the super-resolution movie stacks using MotionCor2 (82) and binned twofold to a calibrated pixel size of $1.054 \AA /$ pix. The defocus values were estimated by Gctf (83) from summed images without dose weighting. Other procedures of cryo-EM data processing were performed within RELION v3.0 $(84,85)$ using the doseweighted micrographs.

For the datasets of the Mediator ${ }^{\mathrm{WT}}$, a subset of $\sim 10,000$ particles were picked by Gautomatch (Zhang, https://www2.mrc-lmb.cam.ac.uk/research/locally-

developed-software/zhang-software/) without reference and subjected to reference-free 2D classification. Selected 2D class averages were low-pass filtered to $20 \AA$ and used as references for automatic particle picking of the whole datasets in RELION resulting in an initial set of 1,594,494 particles for reference-free 2D classification. 702,687 particles were selected from good 2D classes for the initial 3D classification, using a $60 \AA$ low-pass filtered initial model. After several rounds of 2D and 3D classification, 46,190 particles were 3D auto-refined and post-processed, yielding a reconstruction of Mediator ${ }^{\mathrm{WT}}$ at $4.0 \AA$ resolution. We used a local mask 3D classification for the Tail and Head modules, and a masked local refinement followed by the 3D classification. For focused refinement, selected particles were 3D autorefined, CTF refined, Bayesian polished and post-processed, generating reconstructions of the Tail module at $3.6 \AA$ resolution (44,667 particles) and the Head module at $3.4 \AA$ resolution (137,802 particles).

For the datasets of the Mediator ${ }^{\triangle M E D 1-I D R}$, a subset of 10,000 particles were picked by Gautomatch without reference and subjected to reference-free 2D classification. Selected $2 \mathrm{D}$ class averages were low-pass filtered to $20 \AA$ and used as references for automatic particle picking of the whole datasets in RELION resulting in an initial set of 2,069,109 particles for reference-free 2D classification. 1,271,263 particles were selected from good 2D classes for the initial 3D classification, using a $60 \AA$ low-pass filtered initial model. After several rounds of $2 \mathrm{D}$ and $3 \mathrm{D}$ classification, 95,433 particles were 3D auto-refined and post- 
processed, yielding a reconstruction of Mediator complex at $3.5 \AA$ resolution. We used a local mask 3D classification for the modules, and a masked local refinement followed by the 3D classification. For focused refinement, selected particles were 3D auto-refined, CTF refined, Bayesian polished and post-processed, generating reconstructions of the Tail module at $3.2 \AA$ resolution $(98,988$ particles), the Head module at $2.8 \AA$ resolution $(400,082$ particles), the Middle module at $3.7 \AA$ resolution (164,115 particles), MED6 and adjacent subunits at $3.1 \AA$ resolution (107,668 particles), and MED1 and adjacent subunits at $3.6 \AA$ resolution (152,914 particles). For the Mediator with Tail bent $\left(\mathrm{MED}^{\mathrm{B}}\right)$, partial particles were selected to a further global or local mask 3D classification. For the overall structure of $\mathrm{MED}^{\mathrm{B}}$, global 3D classification was performed without mask followed by a 3D autorefinement, yielding a $4.4 \AA(62,871$ particles $)$ reconstruction. For the Tail module in $\mathrm{MED}^{\mathrm{B}}$, local mask 3D classification was performed followed by a $3 \mathrm{D}$ auto-refinement, yielding a $4.5 \AA$ (96,354 particles) reconstruction.

For the dataset of PIC-MED ${ }^{\mathrm{WT}}$, a subset of $\sim 10,000$ particles were picked by Gautomatch without reference and subjected to reference-free 2D classification. Selected 2D class averages were low-pass filtered to $20 \AA$ and used as references for automatic particle picking of the whole datasets in RELION $(84,85)$ resulting in an initial set of 1,318,612 particles for reference-free 2D classification. After removing junk particles through several rounds of $2 \mathrm{D}$ and 3 D classifications, 324,177 particles were selected for further processing. We used a local mask 3D classification around the CTD-Mediator contacts, and a masked local refinement followed by the 3D classification. Selected particles were locally refined in cryoSPARC (86), generating a reconstruction of the CTD-Mediator subcomplex at $4.1 \AA$ resolution (99,145 particles). For overall structure, global 3D classification was performed without mask in $\operatorname{RELION}(84,85)$, yielding three distinct representative maps. Further Nonuniformed refinements were performed, yielding reconstructions of pre-hPIC-MED state at $6.5 \AA$ (22,253 particles), intermediate state at $4.3 \AA$ (32,865 particles), and hPICMED state at $4.2 \AA$ (32,750 particles), respectively.

For the dataset of PIC-MED ${ }^{\triangle M E D 1-I D R}, 1,085,228$ particles were auto-picked by Laplacian-of-Gaussian method in RELION 3.0 $(84,85)$ without reference for reference-free 2D classification. After removing junk particles through several rounds of 2D and 3D classifications, 350,671 particles were selected for further processing. All particles were subjected into 3D classification with soft masks for Pol II, Mediator, CTD-Mediator, and CAK modules, respectively. Selected particles were auto-refined using Local-Refinement in cryoSPARC (86), yielding reconstructions of Pol II at $3.3 \AA$ $(244,108)$, Mediator at $3.8 \AA(163,319)$, CTD-Mediator at 3.7 $\AA(163,319)$ and CAK module at $4.5 \AA(110,227)$. For overall structure, 3D classification was performed with no mask, yielding four maps representing four different conformational states. Further Non-uniformed refinements were performed, yielding reconstructions of pre-hPIC-MED (state1) at $4.1 \AA$, state 2 at $4.0 \AA$, state 3 at $5.2 \AA$, and hPIC-MED (state4) at 4.1 A. For pre-hPIC-MED (state1), Local Refinements were performed with a soft mask for TFIID, TFIIH, TBP, Mediator, and CAK, yielding reconstructions at 7.1 $\AA$, $7.3 \AA, 3.7 \AA, 4.0 \AA$, and $5.7 \AA$, respectively. For hPIC-MED (state4), Local Refinements were performed with a soft mask for TFIID, TFIIH, TBP and Mediator, yielding reconstructions at $8.8 \AA, 8.1 \AA, 3.9 \AA$ and $5.0 \AA$, respectively. Local refinement with a mask for the Tail were also performed and yielded cracked density to show the shape of the Tail.

All reported resolutions were based on the goldstandard Fourier shell correlation $(\mathrm{FSC})=0.143$ criterion. The FSC curves were corrected for the effects of a soft mask with high-resolution noise substitution. All cryo-EM maps were sharpened by cryoSPARC (86) or applying a negative B-factor estimated in RELION $(84,85)$. All the visualization and evaluation of the 3D volume map were performed within UCSF Chimera or UCSF ChimeraX (87), and the local resolution variations were calculated using RELION $(84,85)$.

\section{Model building and structure refinement}

The structural model of Mediator in the $\mathrm{MED}^{\mathrm{E}}$ conformation was built according to the cryo-EM maps of the Mediator complex at $3.5 \AA$ resolution and locally refined cryoEM maps. The structures of human MED23 (PDB: 6H02) (88) and yeast core Mediator (PDB: 5N9J) (89) were used as initial structural templates, which were docked into the cryo-EM maps by rigid-body fitting using UCSF Chimera (87). The structural models were further manually built de novo in COOT (90) and refined in real space using Phenix (91) with secondary structure and geometry restraints using the combined cryo-EM map. Overfitting of the model was monitored by refining the model in one of the two half maps from the gold-standard refinement approach and testing the refined model against the other map (92).

The structural model of $\mathrm{MED}^{\mathrm{B}}$ was built according to the cryo-EM map of the Mediator complex at 4.4 $\AA$ resolution and locally refined cryo-EM map around the Tail at 4.5 $\AA$ resolution. The structural models of modules and domains in $\mathrm{MED}^{\mathrm{E}}$ were docked into the cryo-EM maps followed by rigid-body fitting and manually adjustment in UCSF Chimera (87) and COOT (90).

For the model of PIC-MED, the structures of human Mediator (this study) and hPIC (PDB:7EGB) (45) were used as structural templates, which were docked into the cryoEM maps by rigid-body fitting using UCSF Chimera (87). The structural models were docked according to the cryoEM maps of the overall complex (pre-hPIC-MED at $4.1 \AA$ 
resolution and hPIC-MED at $4.1 \AA$ resolution) and adjusted according to the corresponding locally refined cryo-EM maps in COOT (90). The model of CTD-Mediator and the CAK were built according to the locally refined maps around CTD-Mediator at 3.7 $\AA$ resolution and the CAK at 4.5 $\AA$ resolution in COOT (90). The direction of the two peptides were proposed according to the previously determined crystal structure of CTD-Head (70) and their topological positions relative to Pol II. All the structures were refined in real space using Phenix (91) with secondary structure and geometry restraints. Overfitting of the model was monitored by refining the model in one of the two half maps from the gold-standard refinement approach and testing the refined model against the other map (92).

Statistics of the map reconstruction and model refinement can be found in Data S1. The final models were evaluated using MolProbity (93). All the map and model representations in the figures and movies were prepared by PyMOL (https://pymol.org/) (94), UCSF Chimera (87) or UCSF ChimeraX (95).

\section{Crosslinking MS analyses}

The crosslinking of PIC-MED was performed as previously described (45).

For the crosslinking of Mediator, protein aliquots from the Mono Q peak fractions were dialyzed to completely remove detergent and reduce DTT concentration to $0.5 \mathrm{mM}$. Chemical crosslinking reactions were carried out using crosslinker DSS (Thermo Scientific). Various concentrations of DSS were tested in order to identify an optimal concentration that produce maximal crosslinking efficiency and minimal protein aggregation. For each trial, $0.5 \mu \mathrm{M}$ of Mediator complex was mixed with DSS (from $0.1 \mathrm{mM}$ to 0.25 $\mathrm{mM})$. Crosslinking reactions were carried out at room temperature with shaking at $500 \mathrm{rpm}$ (ThermoMixer) for $1 \mathrm{~h}$. Reactions were quenched by the addition of ammonium bicarbonate (Sigma) to a final concentration of $20 \mathrm{mM}$ and shaking for $20 \mathrm{~min}$ at room temperature. Crosslinking efficiency was visualized by SDS-PAGE. Pre-cooled acetone was added to crosslinked samples. Precipitated proteins were denatured and reduced in $8 \mathrm{M}$ urea, $5 \mathrm{mM}$ TCEP, followed by alkylation with iodoacetamide (Sigma) and trypsin (sequencing grade, Promega) digestion overnight at $37^{\circ} \mathrm{C}$ using a protein to enzyme ratio of 50:1 (w/w). The desalting and peptide enrichment procedure was conducted on a monospin C18 column (GL Sciences) according to the manufacturer's protocol. Then the peptide eluate was dried using centrifugal evaporation and subjected to chromatography. Chromatography was performed as described previously in combination with the FAMIS Pro interface (ThermoFisher) to further improve selectivity, sensitivity and dynamic range. pLink2 was used to analyze the crosslink MS data and annotate the crosslinked peptides (96). Annotated crosslinked sites were represented by xiNET (97) or xVis online server (98).

\section{REFERENCES AND NOTES}

1. M. C. Thomas, C. M. Chiang, The general transcription machinery and general cofactors. Crit. Rev. Biochem. Mol. Biol. 41, 105-178 (2006). doi:10.1080/10409230600648736 Medline

2. R. G. Roeder, The role of general initiation factors in transcription by RNA polymerase II. Trends Biochem. Sci. 21, 327-335 (1996). doi:10.1016/09680004(96)10050-5 Medline

3. L. Zawel, D. Reinberg, Initiation of transcription by RNA polymerase II: A multi-step process. Prog. Nucleic Acid Res. Mol. Biol. 44, 67-108 (1993). doi:10.1016/S0079-6603(08)60217-2 Medline

4. S. R. Albright, R. Tjian, TAFs revisited: More data reveal new twists and confirm old ideas. Gene 242, 1-13 (2000). doi:10.1016/S0378-1119(99)00495-3 Medline

5. S. K. Burley, R. G. Roeder, Biochemistry and structural biology of transcription factor IID (TFIID). Annu. Rev. Biochem. 65, 769-799 (1996). doi:10.1146/annurev.bi.65.070196.004005 Medline

6. B. F. Pugh, R. Tjian, Transcription from a TATA-less promoter requires a multisubunit TFIID complex. Genes Dev. 5, 1935-1945 (1991). doi:10.1101/gad.5.11.1935 Medline

7. L. Warfield, S. Ramachandran, T. Baptista, D. Devys, L. Tora, S. Hahn, Transcription of Nearly All Yeast RNA Polymerase II-Transcribed Genes Is Dependent on Transcription Factor TFIID. Mol. Cell 68, 118-129.e5 (2017). doi:10.1016/j.molcel.2017.08.014 Medline

8. R. Donczew, S. Hahn, Mechanistic Differences in Transcription Initiation at TATALess and TATA-Containing Promoters. Mol. Cell. Biol. 38, e00448-17 (2017). doi:10.1128/MCB.00448-17 Medline

9. R. Donczew, L. Warfield, D. Pacheco, A. Erijman, S. Hahn, Two roles for the yeast transcription coactivator SAGA and a set of genes redundantly regulated by TFIID and SAGA. eLife 9, e50109 (2020). doi:10.7554/eLife.50109 Medline

10. R. Shiekhattar, F. Mermelstein, R. P. Fisher, R. Drapkin, B. Dynlacht, H. C. Wessling, D. O. Morgan, D. Reinberg, Cdk-activating kinase complex is a component of human transcription factor TFIIH. Nature 374, 283-287 (1995). doi:10.1038/374283a0 Medline

11. R. P. Fisher, P. Jin, H. M. Chamberlin, D. O. Morgan, Alternative mechanisms of CAK assembly require an assembly factor or an activating kinase. Cell 83, 47-57 (1995). doi:10.1016/0092-8674(95)90233-3 Medline

12. R. P. Fisher, D. O. Morgan, A novel cyclin associates with M015/CDK7 to form the CDK-activating kinase. Cell 78, 713-724 (1994). doi:10.1016/00928674(94)90535-5 Medline

13. A. Devault, A. M. Martinez, D. Fesquet, J. C. Labbé, N. Morin, J. P. Tassan, E. A. Nigg, J. C. Cavadore, M. Dorée, MAT1 ('menage à trois') a new RING finger protein subunit stabilizing cyclin $\mathrm{H}$-cdk7 complexes in starfish and Xenopus CAK. EMBO J. 14, 5027-5036 (1995). doi:10.1002/j.1460-2075.1995.tb00185.x Medline

14. P. M. Flanagan, R. J. Kelleher 3rd, M. H. Sayre, H. Tschochner, R. D. Kornberg, A mediator required for activation of RNA polymerase II transcription in vitro. Nature 350, 436-438 (1991). doi:10.1038/350436a0 Medline

15. Y. J. Kim, S. Björklund, Y. Li, M. H. Sayre, R. D. Kornberg, A multiprotein mediator of transcriptional activation and its interaction with the $\mathrm{C}$-terminal repeat domain of RNA polymerase II. Cell 77, 599-608 (1994). doj:10.1016/00928674(94)90221-6 Medline

16. C. M. Thompson, A. J. Koleske, D. M. Chao, R. A. Young, A multisubunit complex associated with the RNA polymerase II CTD and TATA-binding protein in yeast. Cell 73, 1361-1375 (1993). doi:10.1016/0092-8674(93)90362-T Medline

17. Z. C. Poss, C. C. Ebmeier, D. J. Taatjes, The Mediator complex and transcription 
regulation. Crit. Rev. Biochem. Mol. Biol. 48, 575-608 (2013). doi:10.3109/10409238.2013.840259 Medline

18. S. Malik, R. G. Roeder, The metazoan Mediator co-activator complex as an integrative hub for transcriptional regulation. Nat. Rev. Genet. 11, 761-772 (2010). doi:10.1038/nrg2901 Medline

19. R. C. Conaway, J. W. Conaway, Function and regulation of the Mediator complex. Curr. Opin. Genet. Dev. 21, 225-230 (2011). doi:10.1016/i.gde.2011.01.013 Medline

20. B. L. Allen, D. J. Taatjes, The Mediator complex: A central integrator of transcription. Nat. Rev. Mol. Cell Biol. 16, 155-166 (2015). doi:10.1038/nrm3951 Medline

21. R. D. Kornberg, Mediator and the mechanism of transcriptional activation. Trends Biochem. Sci. 30, 235-239 (2005). doi:10.1016/j,tibs.2005.03.011 Medline

22. J. Soutourina, Transcription regulation by the Mediator complex. Nat. Rev. Mol. Cell Biol. 19, 262-274 (2018). doi:10.1038/nrm.2017.115 Medline

23. C. Jeronimo, F. Robert, The Mediator Complex: At the Nexus of RNA Polymerase II Transcription. Trends Cell Biol. 27, 765-783 (2017). doi:10.1016/i.tcb.2017.07.001 Medline

24. C. Schiano, A. Casamassimi, M. T. Vietri, M. Rienzo, C. Napoli, The roles of mediator complex in cardiovascular diseases. Biochim. Biophys. Acta 1839, 444-451 (2014). doi:10.1016/j.bbagrm.2014.04.012 Medline

25. J. M. Spaeth, N. H. Kim, T. G. Boyer, Mediator and human disease. Semin. Cell Dev. Biol. 22, 776-787 (2011). doi:10.1016/i.semcdb.2011.07.024 Medline

26. F. J. Asturias, Y. W. Jiang, L. C. Myers, C. M. Gustafsson, R. D. Kornberg, Conserved structures of mediator and RNA polymerase II holoenzyme. Science 283, 985-987 (1999). doi:10.1126/science.283.5404.985 Medline

27. K. L. Tsai, X. Yu, S. Gopalan, T.-C. Chao, Y. Zhang, L. Florens, M. P. Washburn, K. Murakami, R. C. Conaway, J. W. Conaway, F. J. Asturias, Mediator structure and rearrangements required for holoenzyme formation. Nature 544, 196-201 (2017). doi:10.1038/nature21393 Medline

28. K. L. Tsai, C. Tomomori-Sato, S. Sato, R. C. Conaway, J. W. Conaway, F. J. Asturias, Subunit architecture and functional modular rearrangements of the transcriptional mediator complex. Cell 157, 1430-1444 (2014). doi:10.1016/i.cell.2014.05.015 Medline

29. K. Nozawa, T. R. Schneider, P. Cramer, Core Mediator structure at $3.4 \AA$ extends model of transcription initiation complex. Nature 545, 248-251 (2017). doi:10.1038/nature22328 Medline

30. D. J. Taatjes, A. M. Näär, F. Andel 3rd, E. Nogales, R. Tjian, Structure, function, and activator-induced conformations of the CRSP coactivator. Science 295 , 1058-1062 (2002). doi:10.1126/science.1065249 Medline

31. G. Cai, T. Imasaki, Y. Takagi, F. J. Asturias, Mediator structural conservation and implications for the regulation mechanism. Structure 17, 559-567 (2009). doi:10.1016/i.str.2009.01.016 Medline

32. X. Wang, Q. Sun, Z. Ding, J. Ji, J. Wang, X. Kong, J. Yang, G. Cai, Redefining the modular organization of the core Mediator complex. Cell Res. 24, 796-808 (2014). doi:10.1038/cr.2014.64 Medline

33. K. L. Tsai, S. Sato, C. Tomomori-Sato, R. C. Conaway, J. W. Conaway, F. J. Asturias, A conserved Mediator-CDK8 kinase module association regulates Mediator-RNA polymerase II interaction. Nat. Struct. Mol. Biol. 20, 611-619 (2013). doi:10.1038/nsmb.2549 Medline

34. A. M. Näär, D. J. Taatjes, W. Zhai, E. Nogales, R. Tjian, Human CRSP interacts with RNA polymerase II CTD and adopts a specific CTD-bound conformation. Genes Dev. 16, 1339-1344 (2002). doi:10.1101/gad.987602 Medline

35. M. A. Cevher, Y. Shi, D. Li, B. T. Chait, S. Malik, R. G. Roeder, Reconstitution of active human core Mediator complex reveals a critical role of the MED14 subunit. Nat. Struct. Mol. Biol. 21, 1028-1034 (2014). doi:10.1038/nsmb.2914 Medline

36. P. J. Robinson, M. J. Trnka, R. Pellarin, C. H. Greenberg, D. A. Bushnell, R. Davis, A. L. Burlingame, A. Sali, R. D. Kornberg, Molecular architecture of the yeast Mediator complex. eLife 4, e08719 (2015). doi:10.7554/eLife.08719 Medline
37. L. El Khattabi, H. Zhao, J. Kalchschmidt, N. Young, S. Jung, P. Van Blerkom, P. Kieffer-Kwon, K.-R. Kieffer-Kwon, S. Park, X. Wang, J. Krebs, S. Tripathi, N. Sakabe, D. R. Sobreira, S.-C. Huang, S. S. P. Rao, N. Pruett, D. Chauss, E. Sadler, A. Lopez, M. A. Nóbrega, E. L. Aiden, F. J. Asturias, R. Casellas, A Pliable Mediator Acts as a Functional Rather Than an Architectural Bridge between Promoters and Enhancers. Cell 178, 1145-1158.e20 (2019). doi:10.1016/j.cell.2019.07.011 Medline

38. H. Zhao, N. Young, J. Kalchschmidt, J. Lieberman, L. El Khattabi, R. Casellas, F. J. Asturias, Structure of mammalian Mediator complex reveals Tail module architecture and interaction with a conserved core. Nat. Commun. 12, 1355 (2021). doi:10.1038/s41467-021-21601-w Medline

39. Y. He, C. Yan, J. Fang, C. Inouye, R. Tjian, I. Ivanov, E. Nogales, Near-atomic resolution visualization of human transcription promoter opening. Nature 533 359-365 (2016). doi:10.1038/nature17970 Medline

40. S. Schilbach, M. Hantsche, D. Tegunov, C. Dienemann, C. Wigge, H. Urlaub, P. Cramer, Structures of transcription pre-initiation complex with TFIIH and Mediator. Nature 551, 204-209 (2017). doi:10.1038/nature24282 Medline

41. P. J. Robinson, M. J. Trnka, D. A. Bushnell, R. E. Davis, P.-J. Mattei, A. L. Burlingame, R. D. Kornberg, Structure of a Complete Mediator-RNA Polymerase II Pre-Initiation Complex. Cell 166, 1411-1422.e16 (2016). doi:10.1016/j.cell.2016.08.050 Medline

42. R. Abdella, A. Talyzina, S. Chen, C. J. Inouye, R. Tjian, Y. He, Structure of the human Mediator-bound transcription preinitiation complex. Science 372, 52-56 (2021). doi:10.1126/science.abg3074 Medline

43. C. Plaschka, L. Larivière, L. Wenzeck, M. Seizl, M. Hemann, D. Tegunov, E. V. Petrotchenko, C. H. Borchers, W. Baumeister, F. Herzog, E. Villa, P. Cramer, Architecture of the RNA polymerase II-Mediator core initiation complex. Nature 518, 376-380 (2015). doi:10.1038/nature14222 Medline

44. H. Zhang, D.-H. Chen, R. U. H. Mattoo, D. A. Bushnell, Y. Wang, C. Yuan, L. Wang, C. Wang, R. E. Davis, Y. Nie, R. D. Kornberg, Mediator structure and conformation change. Mol. Cell 81, 1781-1788.e4 (2021). doi:10.1016/j.molcel.2021.01.022 Medline

45. X. Chen, Y. Qi, Z. Wu, X. Wang, J. Li, D. Zhao, H. Hou, Y. Li, Z. Yu, W. Liu, M. Wang, Y. Ren, Z. Li, H. Yang, Y. Xu, Structural insights into preinitiation complex assembly on core promoters. Science eaba8490 (2021). doi:10.1126/science.aba8490 Medline

46. M. T. Knuesel, K. D. Meyer, C. Bernecky, D. J. Taatjes, The human CDK8 subcomplex is a molecular switch that controls Mediator coactivator function. Genes Dev. 23, 439-451 (2009). doi:10.1101/gad.1767009 Medline

47. D. Monté, B. Clantin, F. Dewitte, Z. Lens, P. Rucktooa, E. Pardon, J. Steyaert, A. Verger, V. Villeret, Crystal structure of human Mediator subunit MED23. Nat. Commun. 9, 3389 (2018). doi:10.1038/s41467-018-05967-y Medline

48. Y. Li, S. Bjorklund, Y. W. Jiang, Y. J. Kim, W. S. Lane, D. J. Stillman, R. D. Kornberg, Yeast global transcriptional regulators Sin4 and Rgr1 are components of mediator complex/RNA polymerase II holoenzyme. Proc. Natl. Acad. Sci. U.S.A. 92, 10864-10868 (1995). doi:10.1073/pnas.92.24.10864 Medline

49. F. C. Holstege, E. G. Jennings, J. J. Wyrick, T. I. Lee, C. J. Hengartner, M. R. Green, T. R. Golub, E. S. Lander, R. A. Young, Dissecting the regulatory circuitry of a eukaryotic genome. Cell 95, 717-728 (1998). doi:10.1016/S00928674(00)81641-4 Medline

50. C. M. Thompson, R. A. Young, General requirement for RNA polymerase II holoenzymes in vivo. Proc. Natl. Acad. Sci. U.S.A. 92, 4587-4590 (1995). doi:10.1073/pnas.92.10.4587 Medline

51. N. Petrenko, Y. Jin, K. H. Wong, K. Struhl, Evidence that Mediator is essential for Pol II transcription, but is not a required component of the preinitiation complex in vivo. eLife 6, e28447 (2017). doi:10.7554/eLife.28447

52. T. Imasaki, G. Calero, G. Cai, K.-L. Tsai, K. Yamada, F. Cardelli, H. ErdjumentBromage, P. Tempst, I. Berger, G. L. Kornberg, F. J. Asturias, R. D. Kornberg, Y. Takagi, Architecture of the Mediator head module. Nature 475, 240-243 (2011). doi:10.1038/nature10162 Medline 
53. M. Ito, H. J. Okano, R. B. Darnell, R. G. Roeder, The TRAP100 component of the TRAP/Mediator complex is essential in broad transcriptional events and development. EMBO J. 21, 3464-3475 (2002). doi:10.1093/emboj/cdf348 Medline

54. G. Wang, M. A. Balamotis, J. L. Stevens, Y. Yamaguchi, H. Handa, A. J. Berk, Mediator requirement for both recruitment and postrecruitment steps in transcription initiation. Mol. Cell 17, 683-694 (2005). doi:10.1016/i.molcel.2005.02.010 Medline

55. T. Borggrefe, X. Yue, Interactions between subunits of the Mediator complex with gene-specific transcription factors. Semin. Cell Dev. Biol. 22, 759-768 (2011). doi:10.1016/j.semcdb.2011.07.022 Medline

56. W. M. Reeves, S. Hahn, Activator-independent functions of the yeast mediator sin 4 complex in preinitiation complex formation and transcription reinitiation. Mol. Cell. Biol. 23, 349-358 (2003). doi:10.1128/MCB.23.1.349-358.2003 Medline

57. H. Sakurai, T. Fukasawa, Functional connections between mediator components and general transcription factors of Saccharomyces cerevisiae. J. Biol. Chem. 275, 37251-37256 (2000). doi:10.1074/jbc.M004364200 Medline

58. H. Sakurai, Y. J. Kim, T. Ohishi, R. D. Kornberg, T. Fukasawa, The yeast GAL11 protein binds to the transcription factor IIE through GAL11 regions essential for its in vivo function. Proc. Natl. Acad. Sci. U.S.A. 93, 9488-9492 (1996). doi:10.1073/pnas.93.18.9488 Medline

59. S. A. Ansari, M. Ganapathi, J. J. Benschop, F. C. P. Holstege, J. T. Wade, R. H. Morse, Distinct role of Mediator tail module in regulation of SAGA-dependent, TATA-containing genes in yeast. EMBO J. 31, 44-57 (2012). doi:10.1038/emboj.2011.362 Medline

60. M. K. Lim, V. Tang, A. Le Saux, J. Schüller, C. Bongards, N. Lehming, Gal11p dosage-compensates transcriptional activator deletions via Taf14p. J. Mol. Biol. 374, 9-23 (2007). doi:10.1016/i.jmb.2007.09.013 Medline

61. N. Petrenko, Y. Jin, K. H. Wong, K. Struhl, Mediator Undergoes a Compositional Change during Transcriptional Activation. Mol. Cell 64, 443-454 (2016). doi:10.1016/i,molcel.2016.09.015 Medline

62. C. Jeronimo, M.-F. Langelier, A. R. Bataille, J. M. Pascal, B. F. Pugh, F. Robert, Tail and Kinase Modules Differently Regulate Core Mediator Recruitment and Function In Vivo. Mol. Cell 64, 455-466 (2016). doi:10.1016/i.molcel.2016.09.002 Medline

63. K. H. Wong, Y. Jin, K. Struhl, TFIIH phosphorylation of the Pol II CTD stimulates mediator dissociation from the preinitiation complex and promoter escape. Mol. Cell 54, 601-612 (2014). doi:10.1016/i.molcel.2014.03.024 Medline

64. H. J. Baek, S. Malik, J. Qin, R. G. Roeder, Requirement of TRAP/mediator for both activator-independent and activator-dependent transcription in conjunction with TFIID-associated TAF(II)s. Mol. Cell. Biol. 22, 2842-2852 (2002). doi:10.1128/MCB.22.8.2842-2852.2002 Medline

65. S. Malik, H. J. Baek, W. Wu, R. G. Roeder, Structural and functional characterization of PC2 and RNA polymerase II-associated subpopulations of metazoan Mediator. Mol. Cell. Biol. 25, 2117-2129 (2005). doi:10.1128/MCB.25.6.2117-2129.2005 Medline

66. G. Mittler, E. Kremmer, H. T. Timmers, M. Meisterernst, Novel critical role of a human Mediator complex for basal RNA polymerase II transcription. EMBO Rep. 2, 808-813 (2001). doi:10.1093/embo-reports/kve186 Medline

67. F. Coin, V. Oksenych, J. M. Egly, Distinct roles for the XPB/p52 and XPD/p44 subcomplexes of TFIIH in damaged DNA opening during nucleotide excision repair. Mol. Cell 26, 245-256 (2007). doi:10.1016/j.molcel.2007.03.009 Medline

68. F. Coin, L. Proietti De Santis, T. Nardo, O. Zlobinskaya, M. Stefanini, J.-M. Egly, p8/TTD-A as a repair-specific TFIIH subunit. Mol. Cell 21, 215-226 (2006). doi:10.1016/i.molcel.2005.10.024 Medline

69. M. G. Jaeger, B. Schwalb, S. D. Mackowiak, T. Velychko, A. Hanzl, H. Imrichova, M. Brand, B. Agerer, S. Chorn, B. Nabet, F. M. Ferguson, A. C. Müller, A. Bergthaler, N. S. Gray, J. E. Bradner, C. Bock, D. Hnisz, P. Cramer, G. E. Winter, Selective Mediator dependence of cell-type-specifying transcription. Nat. Genet.

\section{2, 719-727 (2020). doi:10.1038/s41588-020-0635-0 Medline}

70. P. J. Robinson, D. A. Bushnell, M. J. Trnka, A. L. Burlingame, R. D. Kornberg, Structure of the mediator head module bound to the carboxy-terminal domain of RNA polymerase II. Proc. Natl. Acad. Sci. U.S.A. 109, 17931-17935 (2012). doi:10.1073/pnas.1215241109 Medline

71. K. D. Meyer, S. C. Lin, C. Bernecky, Y. Gao, D. J. Taatjes, p53 activates transcription by directing structural shifts in Mediator. Nat. Struct. Mol. Biol. 17 753-760 (2010). doi:10.1038/nsmb.1816 Medline

72. W. J. Feaver, J. Q. Svejstrup, N. L. Henry, R. D. Kornberg, Relationship of CDKactivating kinase and RNA polymerase II CTD kinase TFIIH/TFIIK. Cell 79, 11031109 (1994). doi:10.1016/0092-8674(94)90040-X Medline

73. J. Q. Svejstrup, Y. Li, J. Fellows, A. Gnatt, S. Bjorklund, R. D. Kornberg, Evidence for a mediator cycle at the initiation of transcription. Proc. Natl. Acad. Sci. U.S.A. 94, 6075-6078 (1997). doi:10.1073/pnas.94.12.6075 Medline

74. T. M. Søgaard, J. Q. Svejstrup, Hyperphosphorylation of the C-terminal repeat domain of RNA polymerase II facilitates dissociation of its complex with mediator. J. Biol. Chem. 282, 14113-14120 (2007). doi:10.1074/jbc.M701345200 Medline

75. P. Lu, X. C. Bai, D. Ma, T. Xie, C. Yan, L. Sun, G. Yang, Y. Zhao, R. Zhou, S. H. W. Scheres, Y. Shi, Three-dimensional structure of human $Y$-secretase. Nature 512 , 166-170 (2014). doi:10.1038/nature13567 Medline

76. S. M. Vos, L. Farnung, H. Urlaub, P. Cramer, Structure of paused transcription complex Pol II-DSIF-NELF. Nature 560, 601-606 (2018). doi:10.1038/s41586018-0442-2 Medline

77. T. Juven-Gershon, S. Cheng, J. T. Kadonaga, Rational design of a super core promoter that enhances gene expression. Nat. Methods 3, 917-922 (2006). doi:10.1038/nmeth937 Medline

78. R. Fujiwara, K. Murakami, In vitro reconstitution of yeast RNA polymerase II transcription initiation with high efficiency. Methods 159-160, 82-89 (2019). doi:10.1016/i.ymeth.2019.03.016 Medline

79. H. Stark, GraFix: Stabilization of fragile macromolecular complexes for single particle cryo-EM. Methods Enzymol. 481, 109-126 (2010). doi:10.1016/S00766879(10)81005-5 Medline

80. B. Kastner, N. Fischer, M. M. Golas, B. Sander, P. Dube, D. Boehringer, K. Hartmuth, J. Deckert, F. Hauer, E. Wolf, H. Uchtenhagen, H. Urlaub, F. Herzog, J. M. Peters, D. Poerschke, R. Lührmann, H. Stark, GraFix: Sample preparation for single-particle electron cryomicroscopy. Nat. Methods 5, 53-55 (2008). doi:10.1038/nmeth1139 Medline

81. D. N. Mastronarde, Automated electron microscope tomography using robust prediction of specimen movements. J. Struct. Biol. 152, 36-51 (2005). doi:10.1016/j.jsb.2005.07.007 Medline

82. S. Q. Zheng, E. Palovcak, J.-P. Armache, K. A. Verba, Y. Cheng, D. A. Agard, MotionCor2: Anisotropic correction of beam-induced motion for improved cryoelectron microscopy. Nat. Methods 14, 331-332 (2017). doi:10.1038/nmeth.4193 Medline

83. K. Zhang, Gctf: Real-time CTF determination and correction. J. Struct. Biol. 193, 1-12 (2016). doi:10.1016/i.jsb.2015.11.003 Medline

84. J. Zivanov, T. Nakane, B. O. Forsberg, D. Kimanius, W. J. H. Hagen, E. Lindahl, S. H. W. Scheres, New tools for automated high-resolution cryo-EM structure determination in RELION-3. eLife 7, e42166 (2018). doi:10.7554/eLife.42166 Medline

85. S. H. Scheres, RELION: Implementation of a Bayesian approach to cryo-EM structure determination. J. Struct. Biol. 180, 519-530 (2012). doi:10.1016/i.jsb.2012.09.006 Medline

86. A. Punjani, J. L. Rubinstein, D. J. Fleet, M. A. Brubaker, cryoSPARC: Algorithms for rapid unsupervised cryo-EM structure determination. Nat. Methods 14, 290296 (2017). doi:10.1038/nmeth.4169 Medline

87. E. F. Pettersen, T. D. Goddard, C. C. Huang, G. S. Couch, D. M. Greenblatt, E. C. Meng, T. E. Ferrin, UCSF Chimera-A visualization system for exploratory research and analysis. J. Comput. Chem. 25, 1605-1612 (2004). 


\section{doi:10.1002/icc.20084 Medline}

88. H. L. Schubert, J. Wittmeyer, M. M. Kasten, K. Hinata, D. C. Rawling, A. Héroux, B. R. Cairns, C. P. Hill, Structure of an actin-related subcomplex of the SWI/SNF chromatin remodeler. Proc. Natl. Acad. Sci. U.S.A. 110, 3345-3350 (2013). doi:10.1073/pnas.1215379110 Medline

89. K. Luger, A. W. Mäder, R. K. Richmond, D. F. Sargent, T. J. Richmond, Crystal structure of the nucleosome core particle at 2.8 A resolution. Nature $389,251-$ 260 (1997). doi:10.1038/38444 Medline

90. P. Emsley, K. Cowtan, Coot: Model-building tools for molecular graphics. Acta Crystallogr. D 60, 2126-2132 (2004). doi:10.1107/S0907444904019158 Medline

91. P. D. Adams, R. W. Grosse-Kunstleve, L.-W. Hung, T. R. loerger, A. J. McCoy, N. W. Moriarty, R. J. Read, J. C. Sacchettini, N. K. Sauter, T. C. Terwilliger, PHENIX: Building new software for automated crystallographic structure determination. Acta Crystallogr. D 58, 1948-1954 (2002). doi:10.1107/S0907444902016657 Medline

92. A. Amunts, A. Brown, X. C. Bai, J. L. Llácer, T. Hussain, P. Emsley, F. Long, G. Murshudov, S. H. W. Scheres, V. Ramakrishnan, Structure of the yeast mitochondrial large ribosomal subunit. Science 343, 1485-1489 (2014). doi:10.1126/science. 1249410 Medline

93. V. B. Chen, W. B. Arendall 3rd, J. J. Headd, D. A. Keedy, R. M. Immormino, G. J. Kapral, L. W. Murray, J. S. Richardson, D. C. Richardson, MolProbity: All-atom structure validation for macromolecular crystallography. Acta Crystallogr. D 66, 12-21 (2010). doi:10.1107/S0907444909042073 Medline

94. W. L. DeLano, The PyMOL Molecular Graphics System (2002); www.pymol.org.

96. Z. L. Chen, J.-M. Meng, Y. Cao, J.-L. Yin, R.-Q. Fang, S.-B. Fan, C. Liu, W.-F. Zeng, Y.-H. Ding, D. Tan, L. Wu, W.-J. Zhou, H. Chi, R.-X. Sun, M.-Q. Dong, S.-M. He, A high-speed search engine pLink 2 with systematic evaluation for proteome-scale identification of cross-linked peptides. Nat. Commun. 10, 3404 (2019). doi:10.1038/s41467-019-11337-z Medline

97. C. W. Combe, L. Fischer, J. Rappsilber, xiNET: Cross-link network maps with residue resolution. Mol. Cell. Proteomics 14, 1137-1147 (2015). doi:10.1074/mcp.0114.042259 Medline

98. M. Grimm, T. Zimniak, A. Kahraman, F. Herzog, xVis: A web server for the schematic visualization and interpretation of crosslink-derived spatial restraints. Nucleic Acids Res. 43, W362-W369 (2015). doi:10.1093/nar/gkv463 Medline

99. H. Takahashi, T. J. Parmely, S. Sato, C. Tomomori-Sato, C. A. S. Banks, S. E. Kong, H. Szutorisz, S. K. Swanson, S. Martin-Brown, M. P. Washburn, L. Florens, C. W. Seidel, C. Lin, E. R. Smith, A. Shilatifard, R. C. Conaway, J. W. Conaway, Human mediator subunit MED26 functions as a docking site for transcription elongation factors. Cell 146, 92-104 (2011). doi:10.1016/i.cell.2011.06.005 Medline

100. R. Kaufmann, R. Straussberg, H. Mandel, A. Fattal-Valevski, B. Ben-Zeev, A. Naamati, A. Shaag, S. Zenvirt, O. Konen, A. Mimouni-Bloch, W. B. Dobyns, S. Edvardson, O. Pines, O. Elpeleg, Infantile cerebral and cerebellar atrophy is associated with a mutation in the MED17 subunit of the transcription preinitiation mediator complex. Am. J. Hum. Genet. 87, 667-670 (2010). doi:10.1016/i.ajhg.2010.09.016 Medline

101. B. J. Greber, D. B. Toso, J. Fang, E. Nogales, The complete structure of the human TFIIH core complex. eLife 8, e44771 (2019). doi:10.7554/eLife.44771 Medline

102. H. Zhang, D. H. Chen, R. U. H. Mattoo, D. A. Bushnell, Y. Wang, C. Yuan, L. Wang, C. Wang, R. E. Davis, Y. Nie, R. D. Kornberg, Mediator structure and conformation change. Mol. Cell 81, 1781-1788.e4 (2021). Medline

103. F. Yang, B. W. Vought, J. S. Satterlee, A. K. Walker, Z.-Y. Jim Sun, J. L. Watts, R. DeBeaumont, R. M. Saito, S. G. Hyberts, S. Yang, C. Macol, L. Iyer, R. Tjian, S. van den Heuvel, A. C. Hart, G. Wagner, A. M. Näär, An ARC/Mediator subunit required for SREBP control of cholesterol and lipid homeostasis. Nature 442, 700-704 (2006). doi:10.1038/nature04942 Medline

104. A. G. Milbradt, M. Kulkarni, T. Yi, K. Takeuchi, Z.-Y. J. Sun, R. E. Luna, P. Selenko, A. M. Näär, G. Wagner, Structure of the VP16 transactivator target in the
Mediator. Nat. Struct. Mol. Biol. 18, 410-415 (2011). doi:10.1038/nsmb.1999 Medline

105. R. Peruzzini, Z. Lens, A. Verger, F. Dewitte, E. Ferreira, J.-L. Baert, V. Villeret, I. Landrieu, F.-X. Cantrelle, $1 \mathrm{H}, 15 \mathrm{~N}$ and $13 \mathrm{C}$ assignments of the N-terminal domain of the Mediator complex subunit MED26. Biomol. NMR Assign. 10, 233-236 (2016). doi:10.1007/s12104-016-9673-z Medline

106. A. Boija, I. A. Klein, B. R. Sabari, A. Dall'Agnese, E. L. Coffey, A. V. Zamudio, C. H. Li, K. Shrinivas, J. C. Manteiga, N. M. Hannett, B. J. Abraham, L. K. Afeyan, Y. E. Guo, J. K. Rimel, C. B. Fant, J. Schuijers, T. I. Lee, D. J. Taatjes, R. A. Young, Transcription Factors Activate Genes through the Phase-Separation Capacity of Their Activation Domains. Cell 175, 1842-1855.e16 (2018). doi:10.1016/i.cell.2018.10.042 Medline

\section{ACKNOWLEDGMENTS}

We thank Prof. Yu Zhang and Mr. Hongwei Zhang for their help in transcription initiation assay and thank Center of Cryo-Electron Microscopy, Fudan University, Center of Cryo-Electron Microscopy, ShanghaiTec University, Center for Biological Imaging of Institute of Biophysics (IBP) of Chinese Academy of Sciences (CAS), and National Center for Protein Science Shanghai (NCPSS) for the supports on cryo-EM data collection and data analyses and the Biomedical Core Facility, Fudan University for the support on mass spectrometry analyses. Funding: This work was supported by grants from the National key R\&D program of China (2016YFA0500700), the National Natural Science Foundation of China (32030055, 31830107, 31821002), the Shanghai Municipal Science and Technology Major Project (2017SHZDZX01), Shanghai Municipal Science and Technology Commission (19JC1411500), the National Ten-Thousand Talent Program (Y.X.), the National Program for support of Top-Notch Young Professionals (Y.X.), and the Strategic Priority Research Program of the Chinese Academy of Sciences (grant no. XDB08000000). Author contributions: X.Y. and J.L. prepared the samples of Mediator in the apo form for structural analyses with the help from X.C.; Z.W. performed the cryo-EM analyses and the model building of Mediator ${ }^{W T}$, Mediator ${ }^{\triangle M E D 1-I D R}$, and PIC-MED ${ }^{W T}$ with help from W.L.; X.C. and X.W. prepared the samples for structural analyses of PIC$M_{\text {MED }}{ }^{M E D D-I D R}$ and PIC-MEDWT with help from X.Y. and J.L.; Y.Q. performed the cryo-EM analyses and model building of PIC-MED; X.Y. and X.C. performed the XL-MS assays and analyzed the data; Y.X., X.C., X.Y, and J.L. designed the experiments and analyzed the data. Y.X., X.C., X.Y., and J.L. wrote the manuscript; Y.X. supervised the project. Competing interests: Authors declare no competing interests. Data and materials availability: The cryo-EM maps and model coordinates have been deposited to the Electron Microscopy Data Band (EMDB) and Protein Data Bank (PDB). The complexes include PIC-MED $\triangle M E D 1-$ IDR in pre-hPIC-MED state (EMD-31204, PDB: 7ENA), in hPIC-MED state (EMD31207, PDB: 7ENC), in intermediate state2 (EMD-31205) and state3 (EMD31206), and modules (EMD-31208), Mediator ${ }^{\triangle M E D 1-I D R}$ in MEDE (overall, EMD31191, PDB: 7EMF; Head, EMD-31192; Middle, EMD-31193; Tail, EMD-31194; MED1, EMD-31195; MED6, EMD-31196) and MEDB (overall, EMD-31211, PDB: 7ENJ; Tail, EMD-31212) conformations, Mediator ${ }^{W T}$ in MED ${ }^{\mathrm{E}}$ (EMD-31214) and MED $^{B}$ conformations (EMD-31213), and PIC-MEDWT (EMD-31215).

\section{SUPPLEMENTARY MATERIALS}

science.sciencemag.org/cgi/content/full/science.eabg0635/DC1

Supplementary Text

Figs. S1 to S22

Table S1

References (99-106)

MDAR Reproducibility Checklist

Movies S1 to S20

Data S1 to S3

8 December 2020; resubmitted 15 March 2021

Accepted 27 April 2021

Published online 6 May 2021

10.1126/science.abg0635 
A



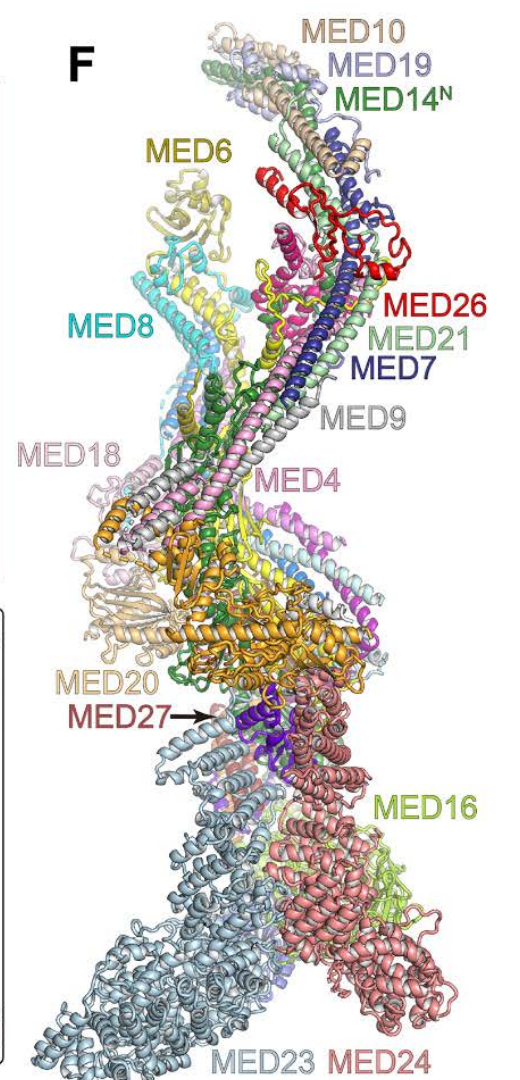

Fig. 1 (preceding page). Overall structure of the human Mediator. (A) Color-coded subunit organization of the human Mediator. The same color scheme is used in all of the figures if not otherwise specified. The unmodeled regions are colored in white and the regions built with poly-alanine chain are indicated with dashed lines. Separated domains are connected by solid lines. (B) A combined cryo-EM map of the human Mediator in two different views. The Middle, Head, and Tail modules are indicated with dashed boxes. The labels of modules and submodules are colored in white and yellow, respectively. ( $C$ to $E$ ) The locally refined cryo-EM maps of Head-Shoulder (C), Middle $(D)$, and Saddle (E). (F) Structural model of Mediator shown in two views as in (B). The $\mathrm{N}$ - and $\mathrm{C}$-terminal regions of MED14 are indicated with MED14 ${ }^{\mathrm{N}}$ and MED14 ${ }^{\mathrm{C}}$, respectively.

Fig. 2 (next page). Structures of the Middle and Head-Shoulder. (A) Structural model of Mediator with MED14 (green) and MED17 (yellow) highlighted and the rest subunits colored in grey. Close-up views of the sequentially arrayed repetitive motifs (RMs) of MED14 and MED17 are shown with $\alpha$-helices colored in lightblue for clarity. (B) Close-up view of the Middle with the major portion of MED1 omitted (shown in Fig. 3A). (C) Structure of the threestranded super-scaffold, in which MED14 (inner), MED17 (central), and the THB (outer) strands are separated by dashed lines. ( $D$ and $E$ ) Close-up views of the binding of THB with MED17 and MED14 (D) and with MED18/20 (E). Unrelated regions are omitted for clarity. 


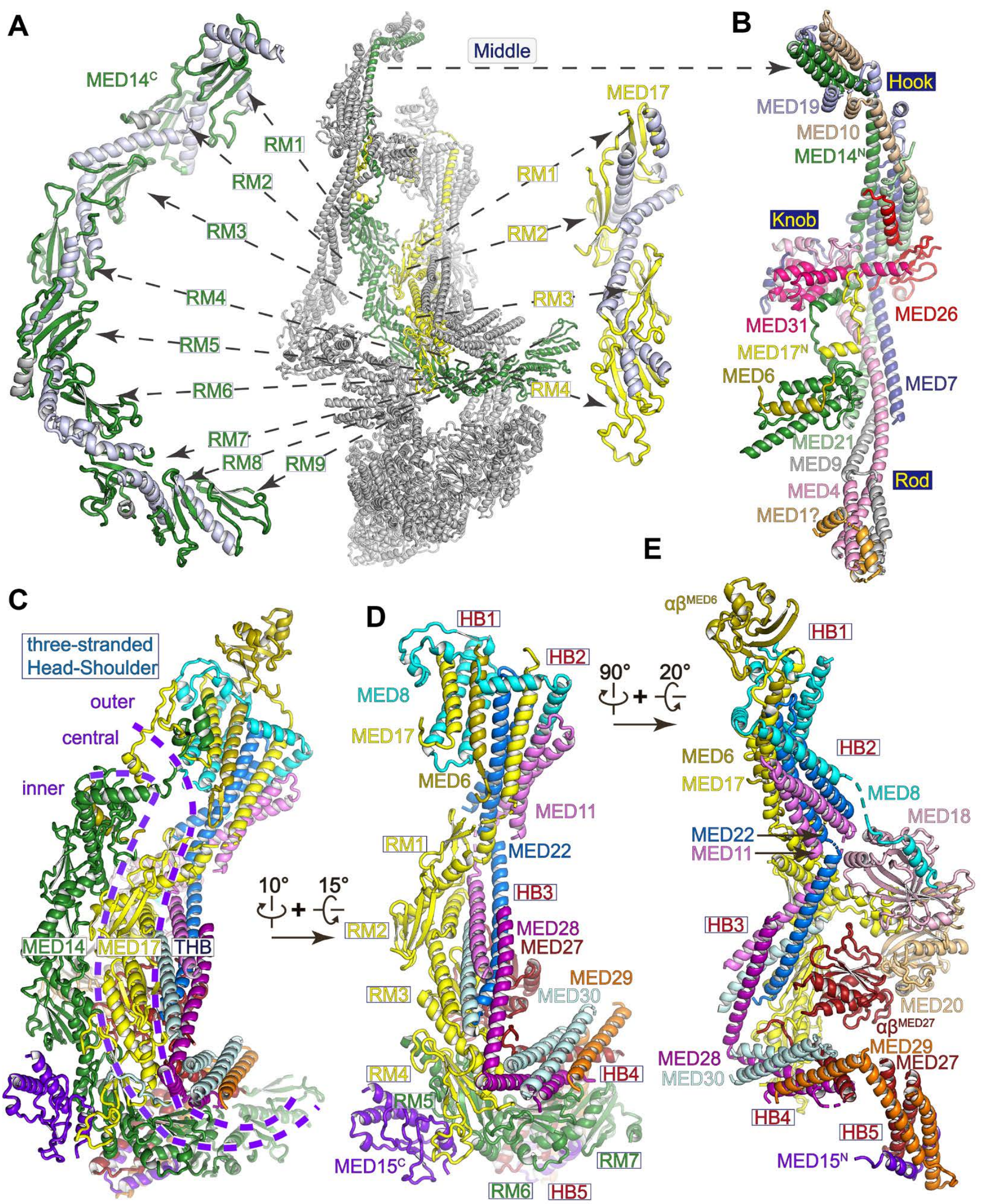




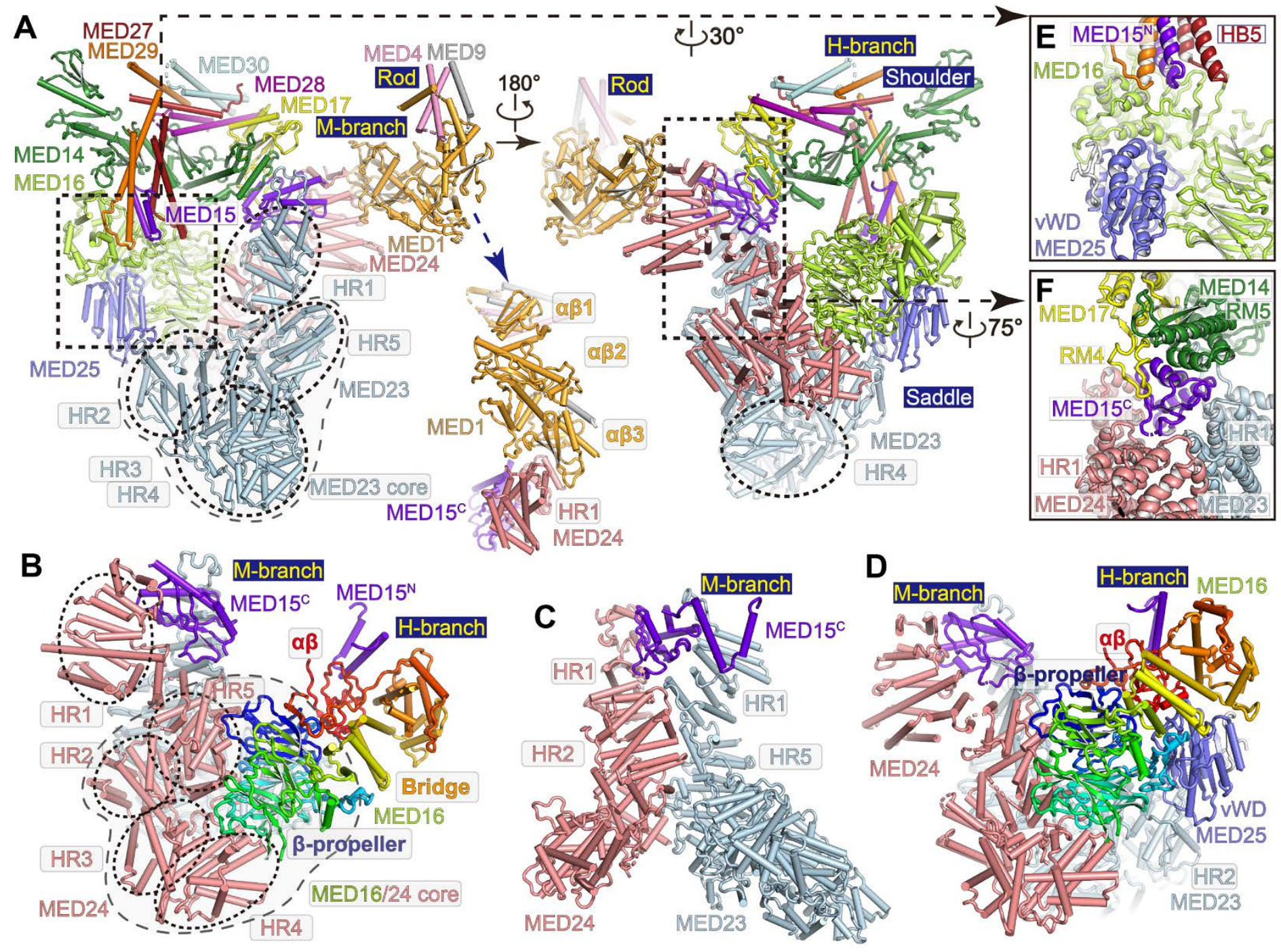

Fig. 3. Structure of the Tail module. (A) Structural model of the Tail in two different views. Two Shoulder-Saddle interfaces are indicated with dashed boxes and shown in close-up views in (E) and (F), respectively. (B to D) Modular organization of subcomplexes in the Saddle. HEAT repeat regions of MED23 and MED24 are highlighted in dashed circles in (A) and (B). Three domains of MED16 ( $\beta$-propeller, Bridge and $\alpha \beta$ domain) are indicated in (B) and (D). The topological arrangement of MED16 is indicated with rainbow-colored scheme with blue to red depicting $N$ terminus to $C$ terminus. 



Fig. 4. Structure of the Mediator in the Tail-bent conformation (MED ${ }^{B}$ ). (A) Comparison of the cryo-EM maps of MED (colored) and MEDE (grey and transparent). Conformational differences in the MED23 core and MED16/24 core are indicated with arrows. Conformational difference in the HR1 ${ }^{\text {MED23 }}$ is shown in close-up view (left panel). MED1 and MED24 are omitted in the left panel for clarity. (B and $C$ ) Structural models of the Saddle in MED ${ }^{E}(B)$ and MED ${ }^{B}(C)$ are shown with Head-Shoulder superimposed as in (A). The MED16/24 core are highlighted in dashed circles. (D) Superimposition of the two models as in (B) and $(C)$ with MED $^{B}$ colored and MEDE in grey. Conformational differences are indicated with arrows. Dashed lines indicate the well-superimposed MED14 and MED1 in (B to D). (E) Comparison of the Saddle structure with MED16/24 core superimposed. Distinct placement of MED23 core indicates modular rearrangement and separation of MED23 and MED24 within the Saddle. 


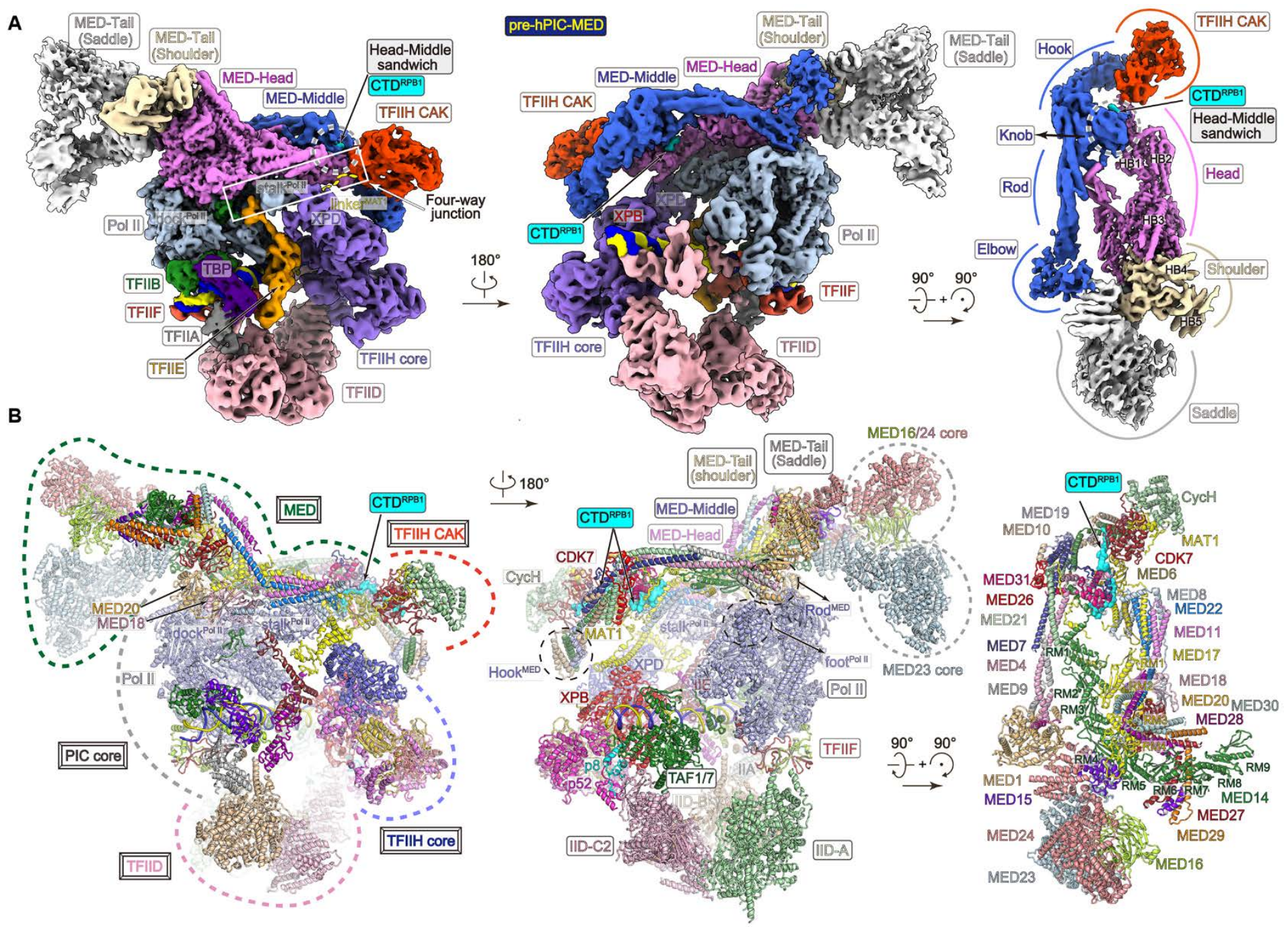

TFIIB $\square$ F-Pol 0.6 MD Pol II TFIIF $\square$ TFIIF $\alpha \square$ TFIIF $\beta \quad$ IID-IIA 1.3 MD $\square$ TFIIA TFIID $\square$ IID-A $\square$ IID-B $\square$ IID-C2 $\square$ IID-C1(TAF1/7) $\square$ TBP

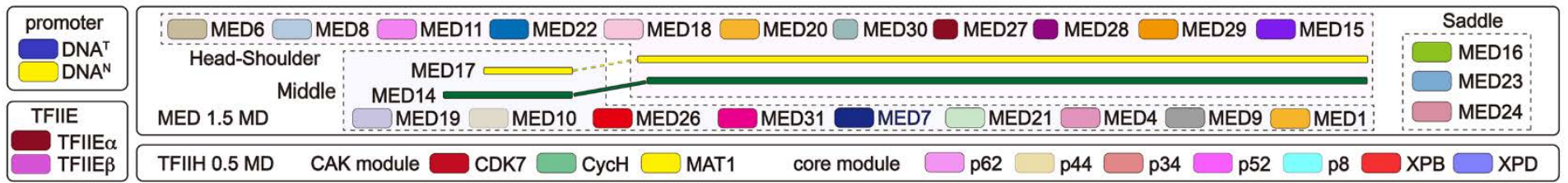

Fig. 5. Structure of the PIC-MED holo-complex. A composite cryo-EM map (A) and structural model (B) of pre-hPICMED shown in three different views. The PIC was omitted in the right panels for clarity. Maps from focused refinements of different modules were combined. Color scheme is indicated below and used throughout all figures of structural models if not elsewhere specified. 



Fig. 6. Binding of PIC leads to concerted reorganization of Mediator. (A to C) Three different views of superimposed cryo-EM maps of pre-hPIC-MED (colored and PIC omitted for clarity) and MEDE (grey and transparent). Structural differences are highlighted with arrows. Similar comparison with MED ${ }^{B}$ is indicated in fig. S16. Positions of three intermolecular contacts shown in (D-F) are indicated with yellow boxes. (D and E) Close-up views of the two well-ordered Saddle domains, the HR1 MED23 $(D)$ and the HR1 ${ }^{\text {MED24 }}(E)$ in pre-hPIC-MED with transparent cryo-EM maps and structural models shown. (F) Superimposed structures of pre-hPIC-MED (colored) and MEDE (grey) with PIC shown in surface and Mediator shown in cartoon. The dashed circle highlights potential clash of the Head with Pol II stalk and MAT1 of TFIIH. (G) Schematic model of Mediator reorganization in PIC-MED (colored) relative to MED ${ }^{B}$ (grey). The Saddle dynamics is indicated with arrow and distinct conformations of the Saddle in MED ${ }^{\mathrm{B}}$ and MED ${ }^{\mathrm{E}}$ are not shown. Two yellow dots represent two fulcra of the two-lever system. The hinge represents the flexible Rod-Elbow tether that connects the two levers. 



E pre-hPIC-MED $\mathbb{I V}$
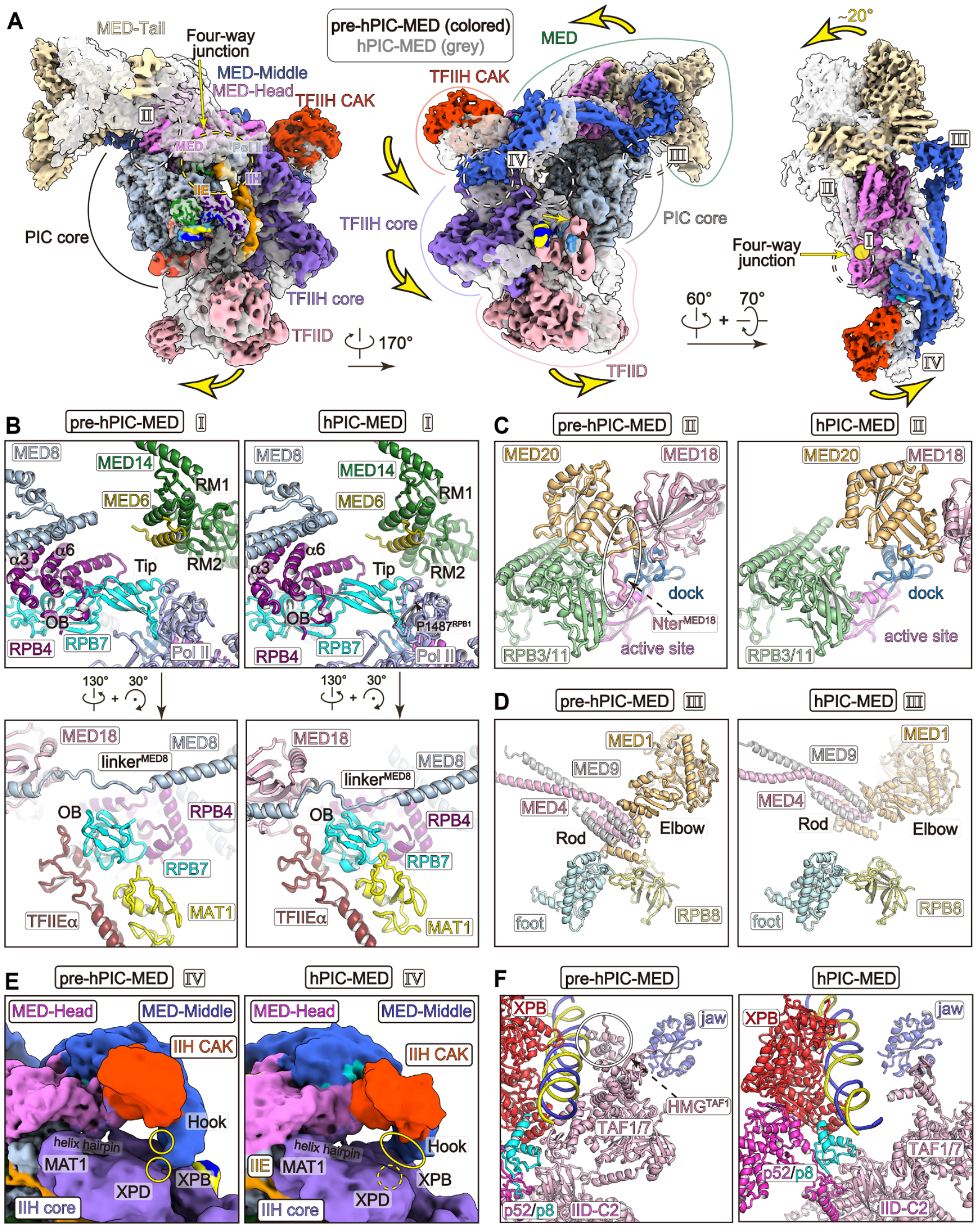

D pre-hPIC-MED 皿
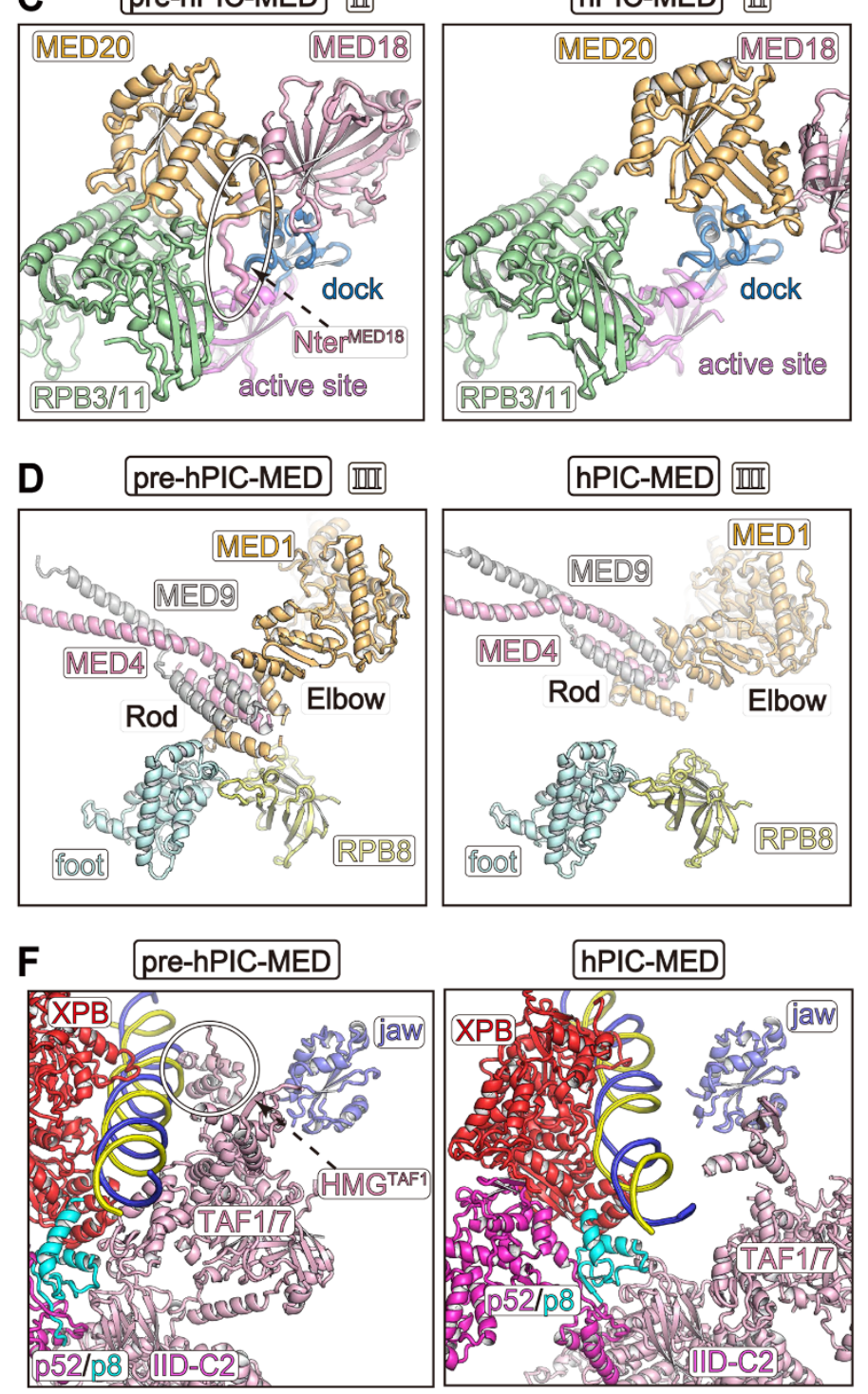
Fig. 7 (preceding page). Dynamics of PIC-Mediator organization. (A) Cryo-EM maps of pre-hPIC-MED (colored) and hPIC-MED (grey and transparent) with Pol II superimposed. Conformational differences are highlighted with arrows. Right panel shows four PIC-Mediator interfaces in pre-hPIC-MED and the axis (yellow dot), around which Mediator rotates. (B to E) Close-up views of PIC-Mediator interface-I/II/III/IV in pre-hPIC-MED (left) and hPICMED (right). The two structures are compared with Pol II superimposed (not shown). Structural models are shown and the related cryo-EM maps are shown in fig. S18, A to D. (C-D) Contacts are apparent at interface-II/III in prehPIC-MED whereas the modules are separated in hPIC-MED. (E) Cryo-EM maps reveal stabilization of the TFIIH core and CAK by Mediator. The Hook binds (solid yellow circles) XPD and XPB in pre-hPIC-MED and XPB in hPICMED. (F) Network interactions among TFIID, downstream promoter, TFIIH core, and Pol II. The related cryo-EM maps are shown in fig. S18E.

Fig. 8 (next page). Mediator brings Pol II CTD into contact with CDK7 and facilitates CTD phosphorylation. (A) Close-up view of intermodular contacts among TFIIE, TFIIH, Pol II CTD, and Head-Middle sandwich of Mediator. Two CTD segments are termed CTD-L and CTD-S. L and S denote longer and shorter segments, repetitively. CTD-L and CTD-S are shown in surface representation and colored in cyan and green, respectively. MED8 is colored in lightblue to distinguish with CTD-L. Domain structure of MAT1 is indicated above the figure. (B to D) Three different views of interactions among Mediator, CTD, and CAK with cryo-EM map shown in transparent surface. The putative CTD-L extension is shown in sticks and its distance to CDK7 active site is indicated in (D). The map in (C) is at higher threshold to show well-separated secondary structure whereas the maps in (B) and (D) are at lower threshold to illustrate relatively weak maps of the MAT1 linker and CTD-L extension. (E) Two CTD-binding sites of the Head-Middle sandwich. The HB1 of the Head and Knob of the Middle are shown in surface and two CTD segments are shown in ribbon. (F) Close-up views of the binding of CTD-L (left panel) and CTD-S (right panel) to the Head-Middle sandwich. The two CTD segments are shown in sticks with cryo-EM maps shown in purple meshes. Putative positions of phosphorylated $\mathrm{S}^{5}$ residues are shown in red balls. (G to I) Residues $\mathrm{Y}^{1}$ of $\mathrm{CTD}^{-}$ LR2/3/4 (R denotes repeat) make contacts with residues of the Head and Middle. $(\mathrm{J})$ Schematic diagram of the network interactions and arrangement of CTD relative to Mediator and CDK7. (K) The proposed CTD-gating model. The central panel represents the structurally observed CTD-sandwiched state. The eCTD repeats are phosphorylated by the HB1-associated CDK7. Two flanking panels illustrate two non-exclusive CTD-gating states. Relative movement between the Head and Middle modules leads to transient separation of the HB1 and Knob on binding site of CTD-L (left panel) or CTD-S (right panel). The CCTD repeats move outward and are brought to CDK7 for phosphorylation. The following CCTD repeats may be sandwiched when Mediator goes back to the CTDsandwiched state. The phosphorylated eCTD may not move back toward Pol II due to the lack of stable interaction with Mediator. The fully-phosphorylated CTD leads to dissociation of Mediator from Pol II and allows for promoter escape of Pol II. 
A




\section{MED17}



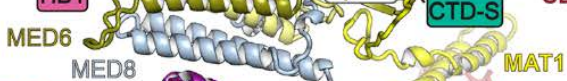
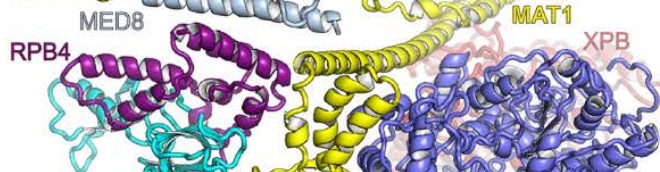

RPB7

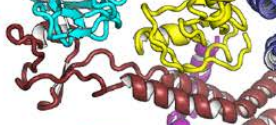

TFIIE $\alpha$ s?

$E$

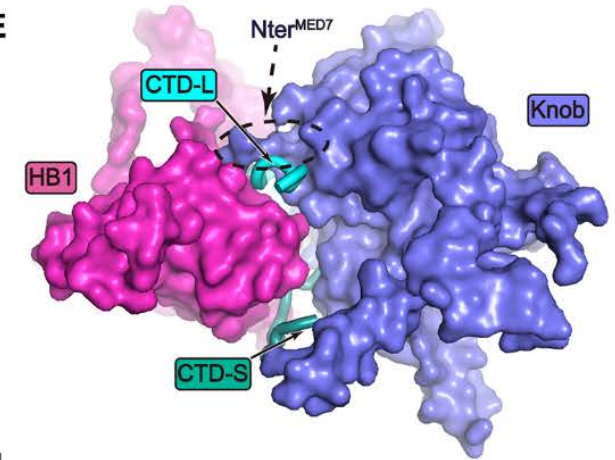

$\mathbf{F}$
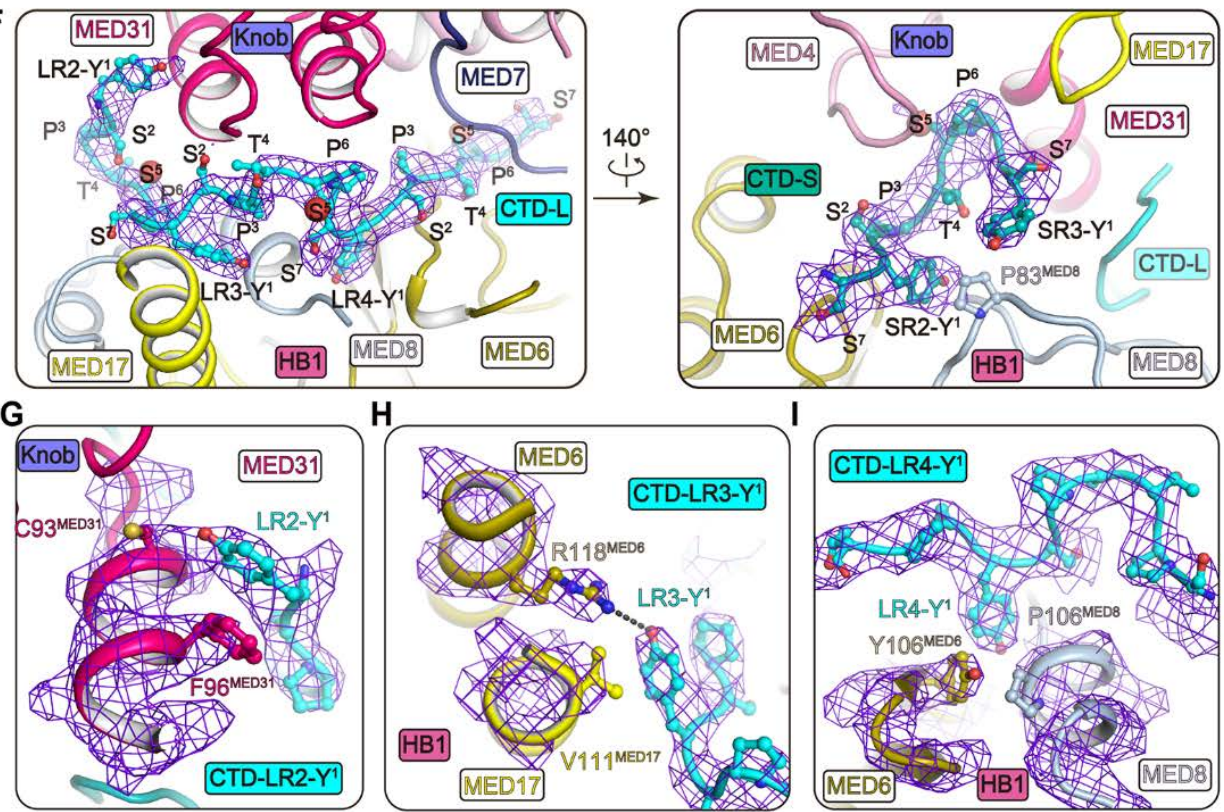

$\mathbf{J}$



LR1 $\frac{\text { LR2 }}{\text { LR3 }}$

- CTD-L extension - - - - -

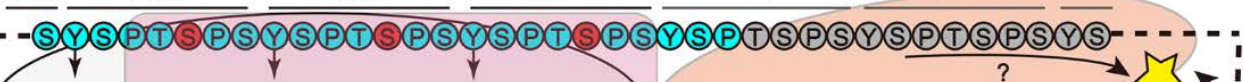




$\overline{\mathrm{SR3}} \mathrm{SR2} \overline{\mathrm{SR}}$

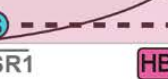

HB1 CTD repeats



K
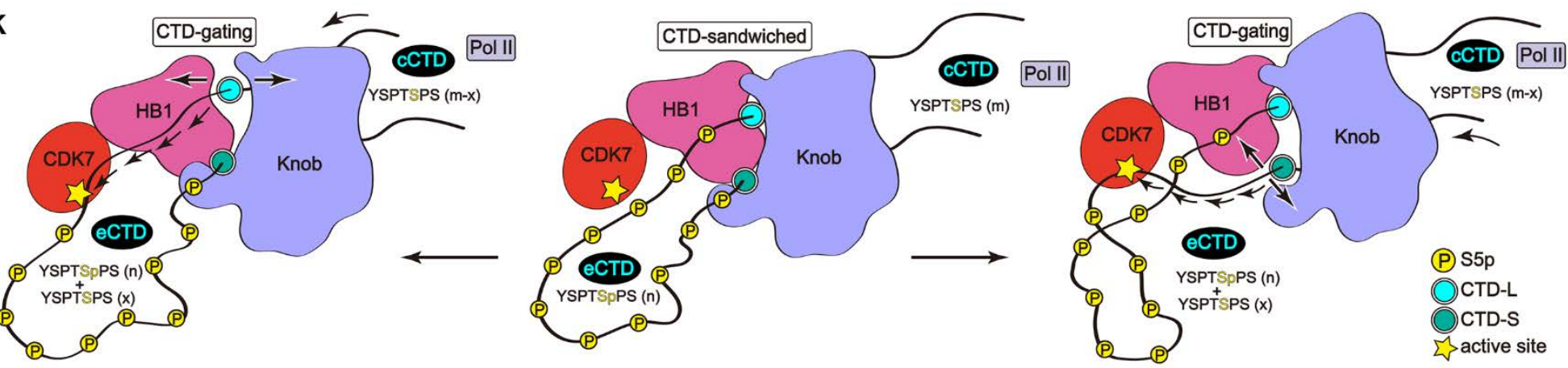